1 Development and evaluation of performance characteristics of timolol-

2 loaded composite ocular films as potential delivery platform for treatment

3

4

5 6 Kent, ME4 4TB

7

$8 \quad{ }^{*}$ Corresponding Author

9 Email: J.S.Boateng@gre.ac.uk; joshboat40@gmail.com

10 Tel: +44(0) 7525630830

11 Fax: +44 (0) 2083319805

12

13

14

15

16

17

18

19

20

21

22

23

\section{of glaucoma}

Mohammad Tighsazzadeh ${ }^{1}$, John C. Mitchell ${ }^{1}$ and Joshua S. Boateng ${ }^{1 *}$.

${ }^{1}$ School of Science, Faculty of Engineering and Science, University of Greenwich, Medway, 


\section{Abstract}

Thin and erodible polymeric films were developed as potential ocular drug delivery systems to increase drug retention on the eye with the aim of improving bioavailability and achieving controlled drug release. Two biocompatible film forming polymers, hyaluronic acid (HA) and hydroxypropyl methylcellulose (HPMC), which are currently used as thickening agents in eye drops were employed. Two different films were prepared (i) as single polymer and (ii) as composite formulations by solvent casting method, incorporating glycerol (GLY) as plasticizer and timolol maleate salt (TM) as model glaucoma drug. After preliminary optimization of transparency and ease of handling, the formulations were further characterized for their physicochemical properties. No indication of significant drug-polymer or polymer-polymer (in composite films) interaction was observed from FTIR results while evaluation by IR mapping revealed uniform distribution of drug throughout the films. Amorphization of TM in the film matrix was confirmed by both DSC and XRD. Swelling studies illustrated remarkable swelling capacity of HA in comparison with HPMC which directly affected the drug release profiles, making HA a suitable polymer for controlled ocular drug delivery. Tensile and mucoadhesion properties confirmed higher elasticity and adhesiveness of HA while HPMC produced stronger films. The effect of sterilization by UV radiation on mechanical properties was also evaluated and showed no significant difference between the sterilized and non-sterilized films. The SEM results confirmed smoothness and homogeneity of film surfaces for all the formulations studied. The in vitro drug dissolution studies showed more extended release profiles of formulations containing HA. Cytotoxicity study (cell viability) using MTT assay on HeLa cells, confirmed that the single polymer and composite films are generally safe for ocular administration. The present work shows excellent film forming ability of HA and HPMC which can be used as single polymer or combined in composite formulations as potential topical ocular drug delivery platform to enhance drug retention on the ocular surface and therefore potential improved bioavailability.

Keywords: Composite films, glaucoma, HPMC, hyaluronic acid, mucoadhesion, ocular delivery, timolol 


\section{Introduction}

Drugs administered using current conventional topical ophthalmic formulations such as eye drops tend to present poor bioavailability due to complex and unique anatomy, physiology and biochemistry of the eye. The sophisticated structure of the eye protects this sensory organ from the external environment, and presents significant limitations to the design of formulations for effective ocular therapy (Zafar et al., 2016).

It has been reported that approximately $4 \%$ of the world's population are visually impaired (Hashemi et al., 2017). This figure could significantly reduce if more efficient ocular dosage forms existed. Currently, instilling topical eye drops is the cheapest and most common method of administering drugs to treat ocular conditions. However, due to existing constraints, less than $5 \%$ of the applied dose is absorbed into the eye. This is because delivery of drug by eye drops is hampered by several anatomical and physiological barriers which limit bioavailability. These include blinking action, tear turnover, nasolacrimal drainage and low permeability of the cornea and/or biological barriers within the eye such as blood aqueous barrier (BAB) or blood retinal barrier (BRB) (Morrison and Khutoryanskiy, 2014). In chronic diseases, such as glaucoma, the drug bioavailability and subsequent therapeutic efficiency obtained with the current conventional dosage forms are considerably low. This results in the need to use other invasive dosage forms such as injections or surgical operations, which are painful and carry a very high risk of damaging the eye tissues and is normally not accepted by patients, especially the younger one. Studies have shown that almost $50 \%$ of glaucoma patients were found to be non-adherent to their currently available medication, $75 \%$ of the time (Aulton and Taylor, 2017).

Glaucoma is a group of eye diseases associated with intraocular hypertension which causes damage to the optic nerve, and if left untreated will lead to blindness. Glaucoma is the second leading cause of blindness worldwide, and it is the world's most common cause of irreversible blindness with 67 million people affected worldwide (Jons et al., 2017). Glaucoma is a progressive optic neuropathy caused by a slow degeneration of retinal ganglion cells and their axons, resulting in a distinct appearance of the optic disc (also known as cupping of the optic nerve) and an associated pattern of visual loss (Weinreb et al., 2014). In glaucoma, the aqueous humor drainage pathway becomes partially or completely blocked, so that fluid cannot easily drain out of the posterior chamber. This causes rapid increase of the pressure within a fixed space of the anterior chamber (intraocular pressure-IOP), causing ocular hypertension which is defined as IOP greater than $21 \mathrm{mmHg}$ (Jons et al., 2017). Most conventional drugs for glaucoma work by either increasing the aqueous humor outflow or reducing its production. 
Timolol (TM), is a non-selective $\beta$-blocker from the family of adrenergic antagonists which reduces IOP by lowering aqueous humor formation and by enhancing the outflow facility (Zafar et al., 2016). All administered ophthalmic formulations require maintenance of visual clarity of the eye, prevention of irritation, infection and inflammation to the eye as well as being able to reach the site of action through the complicated physiological ocular barriers without damaging any healthy tissue (Morrison and Khutoryanskiy, 2014; Gulsen and Chauhan, 2004).

Various attempts have been made in the past few years by different researchers to introduce novel approaches for ocular drug delivery as alternatives to topical eye drops, mainly using polymers to produce thin films, contact lenses, ocular inserts or nanoliposomes (Desai et al., 2018, Boateng and Popescu, 2016, Hui A., 2017, Mehta et al., 2017., Shafie and Rady 2012, Chavda et al., 2016, Jin et al., 2018, Wang et al., 2018). Other studies have reported the synthesis of acryloyl-quaternized poly(2-dimethylamino) ethyl methacrylate) (PDMAEMA) nanogels (Brannigan and Khutoryanskiy, 2017) and polymeric micelles (Mandal et al., 2017). However, the major drawback of micelles and nanogels have been the interference with patient vision and poor transparency of the formulation which was not investigated or mentioned in either studies.

The use of nanoliposomes as ocular delivery system for glaucoma treatment has been reported with maximum drug release occurring in $4 \mathrm{hrs}$ to reduce IOP. However, the IOP started to increase $6 \mathrm{hrs}$ after administration of the liposomes (Jin et al., 2018 and Wang et al., 2018). Desai and co-workers investigated release of TM from hyaluronic acid (HA) based semi-circular ocular inserts implanted onto contact lenses (Desai et al 2018). Despite prolonged release behavior achieved by using HA (one of the polymers being used in this study), essential performance characteristics such as the thickness, mucoadhesion and mobility of the contact lens on the ocular surface were not discussed. The thickness of commercially available contact lenses normally range between 70 - $90 \mu \mathrm{m}$ and therefore addition of $40 \mu \mathrm{m}$ thick insert on top of contact lenses could cause discomfort or mobility limitations on the mucosal surfaces of the eye. In addition, glaucoma is related to insufficient outflow of aqueous humor from posterior chamber of the eye, which is why majority of glaucoma patients experience dry eye and are often prescribed with eye drops to help lubrication of the ocular surface and to prevent damage to healthy eye tissues. Therefore, erodible and thin transparent films comprised of polymers available in the current moisturizing eye drops could potentially overcome the current existing challenges with topical ocular drug delivery in glaucoma. 
Therefore, this study aims to develop novel TM loaded, erodible, and transparent composite thin films using mucoadhesive polymers [hydroxypropyl methylcellulose (HPMC) and hyaluronic acid (HA)], which are currently used as thickening agents in conventional eye drops, as potential drug delivery platforms for glaucoma. Ocular drug delivery using erodible mucoadhesive films offers several advantages over conventional dosage forms such as eye drops and other ocular drug delivery systems (ODDS). These advantages include a significant increase in ocular residence time, prolonged (controlled) release of the drug, accurate dosing, and maintaining lubrication of the eye by moisturizing effect, reduction in administration frequency and increased shelf time.

Hyaluronic acid (HA), also known as hyaluronate, is a naturally derived anionic polysaccharide composed of repeating disaccharide units of N-acetyl-d-glucosamine (1- $\beta-4)$ and d-glucuronic acid (1- $\beta$-3) (Calles et al., 2013). It has been widely used in the pharmaceutical industry over the past few years due to its natural biocompatibility and biodegradability, as well as low level of toxicity and immunogenicity. In addition, HA is a natural component of the eye fluid, as well as connective and epithelium tissues (Papakonstantinou et al., 2012). With its notable adhesive properties, HA is an outstanding choice as a carrier for ocular drug delivery, allowing the loaded drug to be released in a sustained pattern. HPMC is used extensively in the pharmaceutical industry as a film-forming agent, thickener, sustained-release, emulsifying and suspending agent in a wide variety of dosage forms, increasing their dispersity, toughness, sustained release properties and stability (Phadtare et al., 2014). Both HA and HPMC have widespread use in topical eye drops, mainly for their thickening property. Therefore, combining HA and HPMC will produce erodible thin ocular formulations possessing the excellent film forming properties of HPMC and exceptional adhesive characteristic of HA coupled with the biocompatible nature of both polymers with tissues in the human body.

\section{Materials and methods}

\subsection{Materials}

Hydroxypropyl methylcellulose (HPMC) (molecular weight of $1261.45 \mathrm{~g} \mathrm{~mol}^{-1}$ and viscosity of 4,000 cP in water), glucose, gelatin, albumin and timolol maleate (TM) were purchased from Sigma Aldrich, (Gillingham, UK). Hyaluronic acid (HA) (molecular weight $2.6 \times 10^{6} \mathrm{Da}$ ) was purchased from Wisapple, (Beijing, China). Glycerol, sodium bicarbonate, potassium chloride, calcium chloride, sodium chloride, Dulbecco's Modified Eagle's Medium, fetal bovine serum, penicillin-streptomycin, MTT reagent and dimethyl sulfoxide were all purchased from Fisher 
Scientific, (Loughborough, UK).

\subsection{Preparation of TM loaded ocular films}

Films were prepared using hyaluronic acid (HA) and hydroxypropyl methylcellulose (HPMC) as the primary polymers together with glycerol (GLY) as plasticizer (Table 1), using the solvent casting method, and incorporated with TM as model glaucoma drug.

The formulations included single polymer films prepared from $1.0 \%(\mathrm{w} / \mathrm{v}) \mathrm{HA}(\mathrm{F} 1)$ and $1.5 \%(\mathrm{w} / \mathrm{v})$ HPMC (F2) gels, as well as 1\% composite gel with 1:1 ratio of HPMC:HA (F3) and $2 \%$ composite gel with 3:1 ratio of HPMC:HA (F4). All formulations used 2:1 ratio by weight of total polymer: plasticizer following preliminary investigations testing the influence of various concentrations of plasticizer relative to polymer content based on the percent elongation values. Gel preparation was carried out by first dissolving TM $(0.5 \% \mathrm{w} / \mathrm{v})$ in twicedistilled water. Once TM was thoroughly dissolved, appropriate amounts of GLY and the polymer powders (table 1) were added to the TM solution and the mixture was vigorously stirred at room temperature followed by drying in the oven at $40^{\circ} \mathrm{C}$ to obtain the final films.

\subsection{Transparency}

Ocular drugs and delivery systems must be transparent and have zero interference with patient's normal vision. Therefore, the films were evaluated for their clarity and transparency in three different ways. Primary physical transparency was judged by looking through the film to read the numbers on a standard measurement ruler. The evidence and result of this physical appearance judgement was recorded by taking digital images of each labelled film against a clearly numbered ruler. Secondly, the films were randomly shown to five human volunteers, and the participants were asked to score each film from 1 to 5 , with 1 being completely transparent and 5 being completely opaque. The participants were asked to score as individuals in isolation (without any interactions) to avoid possible bias triggered by others' opinion. Finally, the transparency of the films was also measured using UV spectrophotometer to determine the percentage light transmittance for each film at scan speed of $400 \mathrm{~nm} \mathrm{~min}^{-1}$ at three different wavelengths including UVB (290-320 nm), UVA (320-400 nm) and visible light (400-700 nm) (Fuentes et al., 2013).

\subsection{Physicochemical evaluation2.4.1 Thickness and weight}


Film thickness was measured with a digital caliper gauge micrometer and determined at five different locations of each film including four corners and center. To measure the weight of the films, each film was cut into three small disks with $35 \mathrm{~mm}$ diameter and weighed using a digital balance from which average values were calculated. The weight and thickness results were also used in calculation of other physicochemical properties such as tensile and swelling studies respectively.

\subsubsection{Surface $\mathrm{pH}$}

The surface $\mathrm{pH}$ was determined by placing the films in a closed Petri dish left to swell in 0.1 $\mathrm{mL}$ of twice-distilled water at room temperature for $30 \mathrm{~min}$. The insert was removed and placed in close contact with a digital $\mathrm{pH}$ meter to determine the surface $\mathrm{pH}$ (Priya et al 2014).

\subsubsection{Folding endurance}

The folding endurance of the films was determined by folding each film repeatedly at $180^{\circ}$ angle of the plane at the same place until breakage. The films exhibiting folding endurance value of 300 or more were considered to have excellent flexibility (Karki et al 2016).

\subsection{Sterilization}

Exposure to UV radiation was used to sterilize the films prepared in this study. Each film was left in a closed Class II Biological Safety Cabinet (Triple Red, UK) equipped with Philips Germicidal Lamp (Guilford, Surrey, UK). This technique involves exposure of the samples to short-wave ultraviolet (also known as UV-C) within wavelength range of 100-280 nm for 24 hrs. To confirm that the UV radiation did not significantly change the behavior of the films, the tensile and mucoadhesive properties of sterilized films were measured and compared with the corresponding non-sterile films.

\subsection{Swelling index study}

Swelling test was performed using simulated tear fluid (STF) prepared from sodium bicarbonate (192.40 $\left.\mathrm{mg} \mathrm{L}^{-1}\right)$, potassium chloride (111.10 $\left.\mathrm{mg} \mathrm{L}^{-1}\right)$, calcium chloride $(2.30 \mathrm{mg}$ $\left.\mathrm{L}^{-1}\right)$, sodium chloride $\left(672.80 \mathrm{mg} \mathrm{L}^{-1}\right)$, bovine serum albumin $\left(669.00 \mathrm{mg} \mathrm{L}^{-1}\right)$ and glucose ( $\left.2.50 \mathrm{mg} \mathrm{L}^{-1}\right)$ in twice-distilled water. The $\mathrm{pH}$ of STF was set to 7.4 and the fluid temperature was kept at $37{ }^{\circ} \mathrm{C}$ throughout the swelling test. Approximately $2 \mathrm{~mL}$ STF was poured on a previously weighed circular strip of the film (35 mm diameter) and allowed to swell. At specific time intervals, the STF was carefully removed from the film and the sample was weighed again. This was repeated until erosion of the films was observed (determined by the weight loss). The time interval for the measurements were every $2 \mathrm{~min}$ for the first $10 \mathrm{~min}$ and then every $5 \mathrm{~min}$ 
until the films showed signs of erosion. Equation 1 was used to calculate the swelling capacity of each film $(n=3)$.

$$
\text { Swelling Index }=\left[\frac{\left(W_{t}-W_{0}\right)}{W_{0}}\right] \times 100
$$

Where $\mathrm{W}_{\mathrm{t}}$ is weight of swollen film at time $\mathrm{t}$, and $\mathrm{W}_{0}$ is the original film weight at zero time.

\subsection{Tensile properties}

To measure the tensile properties, each film $(n=3)$ was cut into standard dumb-bell shaped strips, which were placed between the texture analyzer (TA) (Stable Micro System, Surrey, UK) grips (probe) for stretching with a gauge length of $30 \mathrm{~mm}$ between each grip and stretched until breaking point, using a $5 \mathrm{~kg}$ load cell with $0.01 \mathrm{~N}$ trigger force. The pre-test speed and the test speed were both set at $1 \mathrm{~mm} \mathrm{sec}^{-1}$, with the post-test speed at $10 \mathrm{~mm} \mathrm{sec}^{-1}$. Equations $(2-4)$ below were used to calculate the tensile strength, elastic modulus and percentage elongation respectively of each film.

Tensile Strength $=\frac{\text { Force at Failure }}{\text { Cross-Sectional Area }}$

Elastic Modulus $=\frac{\text { Slope }}{\text { Crosshead Speed } \times \text { Cross-Sectional Area }}$

Percentage Elongation $=\frac{\text { Increase in Length }(\text { elongation })}{\text { Original Length }} \times 100$

\subsection{In vitro mucoadhesion}

The adhesiveness of the films was also measured by TA using a cylindrical probe $(35 \mathrm{~mm}$ diameter) and $5 \mathrm{~kg}$ load cell against set gelatin gel $(20 \mathrm{~g}, 6.67 \% \mathrm{w} / \mathrm{v})$ as the adhesive surface. To simulate ocular mucosa environment, $500 \mu \mathrm{L}$ of STF was evenly spread on the surface of the gelatin prior to samples contacting the gelatin gel (Momoh et al, 2015). During testing, each film was cut into three $(n=3) 35 \mathrm{~mm}$ circular discs and attached to the end of the cylindrical probe and the probe activated to approach the Petri dish containing the gelatin gel. Each sample disc was left in contact with the moist gelatin surface for $60 \mathrm{sec}$ to ensure complete contact, and then withdrawn at a speed of $1 \mathrm{~mm} \mathrm{~min}^{-1}$ and $0.01 \mathrm{~N}$ trigger force until complete detachment from the gelatin surface. Data obtained from the detachment of the sample was then used to calculate the mucoadhesion properties including peak adhesive force (PAF), total work of adhesion (TWA) and cohesiveness (distance travelled by probe before detachment) of the films. 


\subsection{Attenuated total reflectance (ATR) FTIR spectroscopy}

ATR-FTIR spectra of the films, starting materials and physical mixtures were acquired on a Perkin Elmer Two ATR-FTIR spectrometer (Seer Green, UK). Percentage transmittance (\%T) mode was used in this study with $32 \mathrm{~cm}^{-1}$ resolution with scan speed of 0.2 over wavelength range of $450-4000 \mathrm{~cm}^{-1}$.

\subsection{IR microscopy and imaging}

IR maps and images were collected using a Nicolet iN 10 microscope (Thermo Fisher Scientific, Loughborough, UK) with liquid nitrogen cooled mercury cadmium telluride (MCT) detector and direct sampling with MicroTip ATR, Thermo Fisher Scientific (Loughborough, UK). The data were collected and analyzed by OMNIC Picta software. Different regions of each sample were analyzed by selecting random areas of the film using field view mosaic acquisition with 36 collection points to confirm distribution of TM throughout the film matrix. IR spectra were also obtained at each point together with $2 \mathrm{D}$ and $3 \mathrm{D}$ maps of the film for principal peaks of TM previously identified by ATR-FTIR spectroscopy. The system was set to transmittance mode and wavelength range of $450-4000 \mathrm{~cm}^{-1}$.

\subsection{Thermogravimetric analysis (TGA)}

Residual moisture content of the films was determined using TGA Q5000 SA (Delaware, USA). Samples $(2-5 \mathrm{mg})$ were analyzed at temperature range of $25-300{ }^{\circ} \mathrm{C}$ with heating rate of $10{ }^{\circ} \mathrm{C} \mathrm{min}{ }^{-1}$ under constant stream of dry nitrogen flowing at $50 \mathrm{~mL} \mathrm{~min}{ }^{-1}$ (ElShaer et al., 2016). The plot of weight loss against temperature was obtained and analyzed by $T A$ Instruments Universal Analysis 2000 software to determine the percentage residual moisture content of each sample.

\subsection{Modulated Differential Scanning Calorimetry (MDSC)}

The thermal profiles including the glass transition temperature $\left(\mathrm{T}_{\mathrm{g}}\right)$ were measured for each film as well as pure starting materials using DSC Q2000 (Delaware, USA). Modulated DSC (MDSC) was used for the thin films to allow clearer illustration of glass transition. The DSC thermal analyzer was calibrated using high purity indium by initial cooling from 25 to $-50^{\circ} \mathrm{C}$ at the rate of $-10^{\circ} \mathrm{C} \mathrm{min}^{-1}$. Accurately weighed samples $(3-5 \mathrm{mg}$ ) in pin-holed pans were scanned using the following heating cycles: a) ramp at $5^{\circ} \mathrm{C} \min ^{-1}$ from 25 to $220^{\circ} \mathrm{C}$, b) ramp cooling at rate of $10^{\circ} \mathrm{C} \mathrm{min}^{-1}$ from $220^{\circ} \mathrm{C}$ to zero $0^{\circ} \mathrm{C} \min ^{-1}$ ) ramp at $5^{\circ} \mathrm{C} \mathrm{min}^{-1}$ from zero $0^{\circ} \mathrm{C}$ 


\subsection{Scanning electron microscopy (SEM)}

Surface morphology of the film samples was examined by an ultra-high resolution Hitachi SU8030 SEM (Berkshire, UK). Each sample was sputter coated using chromium for $120 \mathrm{sec}$ at $1 \mathrm{kV}$ and $25 \mathrm{~mA}$ (EmiTech K575X Sputter Coater). Sputter coating of the samples is required prior to SEM imaging to prevent charging of the specimen and to increases the number of secondary electrons that can be detected from the surface of the specimen i.e. increases the signal to noise ratio. Chromium coating was used due to extremely thin nature of the films as well as being more economical than gold coating. Chromium is essentially used in highresolution analysis of thin layers as it produces a very smooth coating, giving exceptionally small grains and an even distribution of chromium nuclei in the coating layer (Stokroos et al., 1997). The SEM images were acquired at an accelerating voltage of $20 \mathrm{kV}$ and working distance of $15 \mathrm{~mm}$, which were then processed with i-scan2000 software. A Hitachi SU8030 cold FEG-SEM with Thermo Fisher Scientific -NORAN System and 7 Ultra-Dry X-ray detectors was used for semi-quantitative energy-dispersive X-ray (EDX) analysis to identify any observed particles on the surface of the films. EDX data were collected at an accelerating voltage of $8 \mathrm{kV}$.

\subsection{X-ray diffraction (XRD)}

The physical form (crystalline or amorphous) of the formulations and starting materials was determined using a D8 Advantage Bruker X-ray diffractometer (Bruker AXS GmbH, Karlsure, Germany) equipped with a Goebel mirror with exit slits of $0.6 \mathrm{~mm}$ and a Lynx eye detector. Since the films are considerably thin, they were folded when placed in the sample holder to ensure maximum amount of sample was available and exposed to X-ray beam for more accurate evaluation. The transmission diffractograms were acquired using a DIFFRAC plus XRD Commander over a diffraction angle range of $5^{\circ}-50^{\circ} 2 \theta$, step size of $0.04^{\circ}$ and scan speed of $0.2 \mathrm{sec}$ per step. The operating conditions during the experiment were $40 \mathrm{kV}$ and $40 \mathrm{~mA}$ with $\mathrm{Cu} \mathrm{K} \alpha$ radiation. The data was processed with EVA software.

\subsection{In vitro drug release}

317 Sampling for in vitro drug release studies was carried out using an automated Gilson FC204

318 fraction collector system (Middleton, USA) coupled with Thermo Fisher SC100 immersion circulators (Loughborough, UK) at $37{ }^{\circ} \mathrm{C}$ and Longer Pump BT100-1L multi-channels 
peristaltic pump (Hebei, China). All formulations were tested simultaneously with STF running through the samples at constant flow rate of $50 \mu \mathrm{L} \mathrm{min}{ }^{-1}$, thus keeping the samples under sink condition throughout the experiment. The entire system as well as the STF bank was kept at 37 ${ }^{\circ} \mathrm{C}$. STF was pumped into the chamber containing the sample from one end and flowed out of the chamber into the collector at the opposite end. This automated sampling technique at the given flow rate was set to mimic the tear turnover in the eye. Once the dissolution medium was collected at specified time intervals, the samples were placed in high-performance liquid chromatography (HPLC) vials and analyzed using an Agilent Technologies 1200 HPLC instrument (Cheshire, UK). Release of TM from the films was detected using a $150 \times 4.6 \mathrm{~mm}$, $5 \mu \mathrm{m}$ reversed phase Spherisorb S5 ODS1 column (Deeside Ind., Clwyd, UK) with methanol (80): water (20): trimethylamine (TEA) (0.2) as the mobile phase, $1 \mathrm{~mL} \mathrm{~min}^{-1}$ flow rate and UV detection at a wavelength of $259 \mathrm{~nm}$ (adapted from Rodriguez et al., 2017).

\subsection{Cytotoxicity and cell viability}

In vitro cytotoxicity evaluation of the films was carried out using HeLa cells supplied by the Tissue Culture Laboratory of the University of Greenwich (Richardson Lab, School of Science, Grenville Building, University of Greenwich at Medway, Kent). In this study, cytotoxicity test was performed by indirect contact of the samples with the cells (Ahmed and Boateng 2018). Cells were cultured in Dulbecco's Modified Eagle's Medium supplemented with $10 \%$ fetal bovine serum and 1\% penicillin-streptomycin (all from Thermo Fisher Scientific, Loughborough, UK). Cells were cultured until 70-80\% confluence and challenged by formulations F1-F4. Films were cut into small disks using a $6 \mathrm{~mm}$ hole punching device and left under UV radiation for $24 \mathrm{hrs}$ for sterilization. The samples were then immersed in $1.5 \mathrm{~mL}$ of complete medium (mentioned above) for $24 \mathrm{hrs}$ in a Heracell 150i $\mathrm{CO}_{2}$ incubator (Thermo Fisher Scientific, Dartford, UK) at $37^{\circ} \mathrm{C}$. The dissolved samples in liquid state were filtered through a $0.2 \mu \mathrm{m}$ filter and the filtrate collected. The cell suspension for the experiment was prepared at a concentration of $1 \times 10^{5}$ cells per $\mathrm{mL}$ and $100 \mu \mathrm{L}$ of cell suspension transferred into designated wells of 96-well tissue-culture microtiter plates. The plates were left in the incubator at $37{ }^{\circ} \mathrm{C}$ in $5 \%$ (v/v) $\mathrm{CO}_{2}$ for 24,48 and $72 \mathrm{hrs}$. Evaluation of the in vitro cytotoxicity of the films based on cell viability was determined by the 3-(4,5dimethylthiazol-2-yl)-2,5-diphenyltetrazolium bromide (MTT) assay. At each time point (i.e. 24, 48 and $72 \mathrm{hrs)} 10 \mu \mathrm{L}$ of MTT reagent (Thermo Fisher Scientific, Loughborough, UK) was added to each well including blank and controls and left in the incubator for an additional 
$4 \mathrm{hrs}$. Media was then completely removed from all wells and replaced with $100 \mu \mathrm{L}$ of dimethyl sulfoxide (DMSO) (Thermo Fisher Scientific, Loughborough, UK). The plates were returned to the incubator for $30 \mathrm{~min}$ and the absorbance recorded at $520 \mathrm{~nm}$ by a microtiter plate reader (Multiskan FC, Thermo Fisher Scientific, Loughborough, UK) equipped with SkanIt for Multiskan FC 3.1 software (Thermo Scientific, Loughborough, UK). Every experiment was carried out in triplicates $(n=3)$ and the percentage cell viability was calculated using equation 5;

Cell viability $(\%)=\frac{A t-A b}{A c-A b} \times 100$

Where $A t, A b$ and $A c$ are the absorbance of tested samples, blank (medium only) and negative control (untreated cells) respectively.

\subsection{Statistical analysis}

Statistical analysis of quantitative data in this study was performed using one-way analysis of variance (ANOVA) and t-test. The level of significance chosen was 0.05 with $p$ values below 0.05 considered significant and measurements are presented as mean ( \pm standard deviation).

\section{Results and discussion}

Optimized ocular films prepared in this study using solvent casting method confirmed the film forming abilities of HA and HPMC and their potential benefits in ocular drug delivery. The initial visual examination demonstrated the ability of both polymers to produce strong transparent films which were flexible and easy to handle both as single polymer and composite formulations. The visual assessment of transparency showed optimum results for all formulations with F4 being slightly cloudy yet transparent overall, as shown by legibility of the underlying ruler in figure 1.

\subsection{Transparency}

Transparency of the films was further confirmed by measuring the light transmittance using UV spectroscopy, and the overall response is illustrated in figure 2. As can be observed in the figure, almost all the formulations showed light transmission values above $80 \%$ in the visible light $(400-700 \mathrm{~nm})$ region. The lower percentage light transmittance of F4, $75.87 \pm$ 4.55 , is suspected to be due to higher concentration of polymer in that formulation (table 1). Higher polymer concentration in F4 increases density of materials in the film (i.e. higher weight and thickness) which subsequently affected the transparency of this film as light transmission is directly affected by density of materials. This slight opacity of F4 in transmission of visible 
light was also visually observable by looking through the film as shown in figure 1 .

Further, transparency of the films was investigated by means of an in vivo human visual examination survey. The result for the volunteers and their score for each film confirms the results from the two previous transparency measurements discussed above. The average score for formulation F1 to F4 was 1.0, 1.0, 1.2 and 1.6 respectively. Though F4 was considered transparent, the degree of transparency was also judged by the volunteers to be slightly lower than other formulations.

\subsection{Physicochemical evaluation}

\subsubsection{Weight and thickness}

Weight and thickness of the films increased with increase in polymer concentration. Both weight and thickness play key roles in certain characteristics such as swelling, tensile properties and drug release. Generally, the weight and thickness results were found within the standard range of commercially available contact lenses with F4 having the maximum accepted (Johnson \& Johnson Acuvue standards) thickness of $0.09 \mathrm{~mm}$ (table 2). The higher thickness value of F4 is again due to the presence of higher concentration of polymer $(\mathrm{w} / \mathrm{v})$ in comparison with the other formulations (table 1).

\subsubsection{Surface $p H$}

The optimum $\mathrm{pH}$ of an ocular formulation is $7.2 \pm 0.02$, however, the buffering capacity of the tears allows the eyes to tolerate $\mathrm{pH}$ values in the 3.5-8.5 range (USP Forum 35-5; Imperiale et al., 2018). The $\mathrm{pH}$ range of the formulations in this study was $5.97 \pm(0.08)$ to $6.46( \pm 0.05)$ (table 2) which is within the accepted $\mathrm{pH}$ range for topical ocular administration and therefore not expected to cause any irritation when applied to the eyes.

\subsubsection{Folding endurance}

The flexibility of the polymeric thin films was measured with respect to their folding endurance. The results suggested excellent flexibility, with each film formulation remaining intact after more than 300 folding repeats. The flexibility of the films is critically important considering the fact that the ocular films will be handled by patients and need to be administered without breakage as well as not causing contact irritation or damage to healthy eye tissues due to brittleness.

\subsection{Swelling index study}

The swelling capacity was investigated to evaluate the hydrophilicity, hydration and erosion of the films, and the results are presented in figure 3. Both F1 and F3 swelled rapidly in the first 
42010 min with swelling index values of $2039.6 \%$ and $1767.9 \%$ respectively. Swelling of F2 and 421 F4 films after 40 min was significantly lower $(p<0.05)$ than F1 and F3. Formulations containing higher ratio of HPMC i.e. F2 and F4 showed an overall lower swelling capacity compared to F1 containing only HA or F3 with equal ratios of HA:HPMC.

The degree of swelling depends on the rate of penetration of fluid into the polymer matrix and the matrix resistance to movement of the water molecules within it and eventual erosion of the matrix. High molecular weight polymers such as $\mathrm{HPMC}\left(\mathrm{C}_{56} \mathrm{H}_{108} \mathrm{O}_{30}\right)$ normally produce physically stronger film sheets due to shorter distance between the polymer chains. In addition, due to presence of nitrogen in $\mathrm{HA}\left(\mathrm{C}_{14} \mathrm{H}_{21} \mathrm{NO}_{11}\right)$, an additional electronegative element in the repeating monomer, it forms secondary hydrogen bonds (in red, figure 4) which results in a stronger matrix despite the longer distance between polymer chains.

The molecular characteristics of the polymers play an important role in swelling capacity, erosion and hence the release of the drug from the film matrix. Though HPMC forms stronger films, the chemical bonds between the polymer chains are only supported by hydrogen bonding between its hydroxyl groups, which allows easy penetration of water inside the matrix, and even easier polymer chain disentanglement and relaxation. The polymer chains in HA containing films (F1, F3 and F4), on the other hand, are held more strongly by two types of hydrogen bonding, involving the $\mathrm{N}-\mathrm{H}$ and O$\mathrm{H}$ groups which hold the matrix more tightly together despite the increase in distance between the polymer chains upon penetration of water. This results in ability to absorb and hold on to more water (i.e. higher swelling index) and to also delay the disentanglement of polymer chains and ultimately the release of any incorporated substances from the film matrix. This ability of HA to hold more water (confirmed by TGA results) also enhances the flexibility of HA (i.e. F1, F3 and F4) as water itself acts as a plasticizer within the film. In addition, HA and HPMC are completely amorphous materials and are easier to hydrate and erode compared to crystalline materials due to their irregular polymer-polymer bonds. It was reported by Adel and ElKasabgy that the presence of plasticizer in formulations affects the percentage of moisture absorbed. In addition to the water content, the films also contained plasticizer (i.e. GLY) which enhances flexibility of the films even more by reducing the glass transition temperature $\left(T_{g}\right)$ (Adel and ElKasabgy, 2014). In general, polymers with high $T_{g}$ often suffer from brittle behavior and low processability.

\subsection{Tensile properties}

450 The results obtained from stress/strain curve was used to calculate mechanical characteristics of the films which are presented in table 3. The tensile strength of formulations F1-F4 was within the range of $39.98 \pm 11.71$ to $166.33 \pm 21.19 \mathrm{Nmm}^{-2}$. The elastic modulus of all films 
elongation $51.66 \pm 0.81$ while $\mathrm{F} 1$ showed the highest value of $72.53 \pm 6.37$. Generally, all films displayed a uniform correlation between stress and strain and produced typical curve of a material with ideal elastic behavior (Bhamra and Tighe, 2017).

Tensile strength and elastic modulus results revealed formulations containing higher ratio of HPMC i.e. F2 and F4 produced considerably stronger films compared to F1 and F3. However, F1 (containing HA only) produced the most elastic film confirmed by the highest \% elongation value $72.53 \pm 6.37$ amongst all formulations. Tensile strength and \% elongation values were compared to that from Priya and co-workers (2014) study, where they produced films using HPMC, PVP and propylene glycol. Generally, both tensile strength and \% elongation values of F1-F4 were higher than all the formulations in that study. Interestingly, the tensile strength of F2 and F4 were considerably higher than all films produced in the study by Priya et al. Further, the folding endurance results in their study, showed that the maximum folding endurance was 29 , which indicates the films were considerably brittle compared to F1F4 in this study with folding endurance of $>300$.

Water content of the films was considered to affect the elasticity and hence elongation values due to plasticizing effects of water within the film matrices. Tranoudis and Efron (2004) revealed no significant relationship between water content and mechanical properties of soft contact lenses. However, contact lenses are made from non-erodible hydrogels where presence of water does not necessarily influence the physical state of the hydrogel because the polymerpolymer bonds are not affected by water molecules. In the case of the thin erodible ocular films prepared in this study, water content (confirmed by TGA) plays a major role in elasticity of films, as the polymer chains interactions are susceptible to water molecules as was also confirmed by swelling results. Another significant factor is polymer concentration which had direct correlation with weight, thickness and density of the films (table 2) and ultimately the mechanical properties of the films.

Strength and flexibility are both essential in ocular films because appropriate strength helps to prevent tearing due to stress generated by blinking action of the eye, and flexibility for ease of handling and application by the patient and to avoid irritation to the eye (Jethava et al., 2014). Therefore, use of the two polymers within the composite films combines the physical advantages of each polymers i.e. strength of HPMC and flexibility of HA.

\subsection{In vitro mucoadhesion}

Evaluation of mucoadhesion properties of formulations F1-F4 are summarized in table 4. Formulation F2, containing HPMC only, showed the lowest stickiness (PAF), TWA and 
cohesiveness amongst all the films. These values almost doubled in F1 (HA only formulation). Interestingly, the combination of the two polymers in the composite films (F3 and F4) enhanced PAF and TWA values compared to single polymer formulations. However, the difference in mucoadhesion properties of the films was not statistically significant $(p>0.05)$. F3 showed the most optimum mucoadhesion properties amongst all formulations (table 4).

In vivo adhesion of films to mucosal surfaces of the eye such as cornea or cul-de-sac is due to interaction of the polymeric thin films with the tear fluid, or more specifically the lipidrich layer (outermost layer) of the tear film which is partially composed of meibum produced by fully differentiated meibocytes in the holocrine meibomian glands. Despite variations in the published compositions, sterol esters and wax esters seem to be the most abundant lipid species in the meibum (Rantamäki et al., 2011). Although meibum lipids have been studied widely, comprehensive tear fluid lipidomic studies are lacking. However, in a neutral medium, the mucin molecules are negatively charged $(\mathrm{pKa}-2.6)$ and behave as anionic polyelectrolytes, forming a weak viscoelastic gel which consists of a network of linear, flexible and random coil molecules. Polymer-mucin interactions include chain interlocking, conformational changes and non-covalent bond formation (Chavda et al., 2016). Polymers such as HA and HPMC have functional groups that are able to form hydrogen bonds and the polymer chain are flexible enough to form as many intermolecular bonds as possible.

The enhanced mucoadhesion properties due to presence of $\mathrm{HA}$ is attributed to remarkable wettability and hydration of HA exhibiting strong non-covalent intermolecular interaction with the gelatin substrate which was used to mimic the mucosal surface of the eye (Ayensu et al., 2012; Nowak et al., 2015). Though the lipid-rich layer containing meibum (in an in vivo setting) was absent, spreading STF on the gelatin surface allowed us to simulate the ocular mucosa surface for in vitro mucoadhesion studies where the salts present in STF act as charged polyelectrolytes. However, the presence of esters in meibum could potentially increase the number of hydrogen bonding and Van der Waals interactions which would further enhance the adhesion of the films on the model mucosal surface. Once again, the presence of amine group in HA creates secondary intermolecular hydrogen bond interaction with chains of the hydrated gelatin surface which acts as additional force compared to HPMC, which only relies on hydrogen bonds of the hydroxyl group. In general, the initial stages of mucoadhesion involves physical contact of the film and the mucosal surface which results in hydration of the polymer, leading to formation of physical entanglement between the polymer and the gelatin substrate and establishing adhesive forces between the two interacting surfaces. Presence of salts (in STF) have also been reported amongst factors affecting the mucoadhesion properties 
522 of polymer-based systems for topical mucosal applications (Khan et al., 2016).

523 3.6. Sterilization

524 It is a mandatory requirement for every formulation designed for topical ocular administration 525 to be completely sterile and free of microorganisms to ensure their safety for patients. The most 526 reliable sterilization method in the industry, based on International Pharmacopoeia (8th edition, 527 2018, section 5.8), is exposure to saturated steam under pressure in an autoclave. This method 528 is used to sterilize commercially produced TM eye drops. Other sterilization methods include gamma and ultraviolet (UV) radiation (Alariqi et al., 2016). During this study, the research team had no access to gamma radiation, and steam autoclave could not be used because of swelling of the hydrophilic films; therefore, UV radiation technique was used for sterilization.

Desai and co-workers (2018) attempted the autoclave sterilization approach and observed loss of dosage form during the sterilization process which subsequently affected the amount of drug available during drug release studies. They subsequently concluded that use of UV radiation an appropriate sterilization approach for polymer based ocular inserts (Desai et al., 2018). Sterilization by UV radiation is a simple, effective and cost-efficient method that has been shown to preserve biocompatibility of the sterilized materials. Short-wave UV irradiation (100-280 nm) causes disruption of DNA-based pairing leading to inactivation of bacteria, viruses and protozoa allowing sterilization of the samples (Rastogi et al., 2010). Though sterilization is aimed to improve the biocompatibility of the formulation, it can cause adverse effect on the performance of formulations and certain physicochemical properties, such as tensile strength and elongation (Galante et al., 2018 and Yeh et al., 2011). Therefore, the tensile and mucoadhesion properties of the sterilized ocular films were analyzed to investigate the potential effects of UV radiation on these mechanical characteristics of the films. Sterilization by UV radiation showed no significant effect $(p>0.05)$ on physical and mechanical properties of the films. The variation observed in the post-sterilization results showed no consistent pattern, and when compared to non-sterile films the difference was not statistically significant $(p>0.05)$. Assessment of results for tensile properties of single polymer formulations (F1 and F2) revealed only slight changes in the values after sterilization. Tensile strength value $\left(\mathrm{N} / \mathrm{mm}^{2}\right)$ of $\mathrm{F} 1$ reduced from $21.01 \pm 4.64$ to $18.66 \pm 6.91$ while its \% elongation value increased from $0.98 \pm 0.25$ to $2.18 \pm 1.52$. In composite formulations, (F3 and F4), the $\%$ elongation decreased in both formulations from $51.66 \pm 0.81$ to $49.91 \pm 1.15$ and from 51.19 \pm 12.33 to $49.41 \pm 3.88$, respectively. Tensile strength and elastic modulus of the composite F3 and F4 films also showed no consistent pattern. Mucoadhesion results revealed general increase in stickiness of the films after sterilization, indicated by higher PAF values. Cohesiveness of 
single polymer F1 and F2 films increased after sterilization while the composite F3 and F4 formulations showed reduction in cohesiveness.

Taking standard deviation values into consideration, together with statistical analysis, the difference in results before and after sterilization is statistically insignificant. The nonsignificant differences observed is suspected to be due to exposure of the films to air for $24 \mathrm{hrs}$ during sterilization which can cause alterations in moisture content of the films and hence slight difference in mechanical properties. Loss of water can increase the tensile properties as the distance between the polymer chains reduces, while reducing the elasticity of the films, since water has known plasticizing effects.

\subsection{ATR FT-IR spectroscopy}

FTIR analysis is often used to show compatibility and interactions between different excipients within a formulation. Secondary interactions such as hydrogen bonding and van der Waals (induced dipoles) tend to increase the stability of structures which can be detected by shifts in wavelength in the FTIR spectra (Mehta et al., 2017). The FTIR spectra of the films (F1-F4), pure polymer powders and pure TM powder were assessed and compared to evaluate the drugpolymer interaction in all formulations (figure 5) and to confirm the stability of TM in the film matrices. The FTIR spectrum of TM pure powder showed principal peaks at $2976 \mathrm{~cm}^{-1}$ and $2892 \mathrm{~cm}^{-1}$ corresponding to stretching of hydroxyl group. The spectra of HA and HPMC powders are also presented in figure 5(b) together with the spectra of all physical mixtures. In addition, the absorption band due to bending of the amine group was observed as a shoulder to the main peak at $1698 \mathrm{~cm}^{-1}$ as shown in figure $5 \mathrm{~b}$ below. The spectra of the physical mixture revealed no considerable changes when compared to FTIR peaks of TM, confirming no major physical interactions within the mixture. Evidently the drug-polymer interaction was also absent in the films as the principal peaks of incorporated TM in the films appeared in similar regions in the spectra of formulations F1-F4 with no major shifts observed, as shown in figure $5(a)$.

For instance, in $\mathrm{F} 1$, the peaks at $2976 \mathrm{~cm}^{-1}$ and $2892 \mathrm{~cm}^{-1}$ due to stretching of $-\mathrm{OH}$ group in TM appeared at $2928 \mathrm{~cm}^{-1}$ and $2880 \mathrm{~cm}^{-1}$ adjacent to the $-\mathrm{OH}$ bend of the films which is due to water content available in formulations (confirmed by TGA results). Other principal peak of TM at $1698 \mathrm{~cm}^{-1}$ due to bending of $-\mathrm{NH}$ group in TM also appeared in the spectra of all formulations with negligible shifts in wavelength. The evaluation of ATR-FTIR results confirms the stability of TM in the formulations developed in this study with no major drugpolymer interaction. 


\subsection{IR microscopy and imaging}

592 Distribution of TM in formulations F1-F4 was investigated and confirmed by mapping the availability of TM across the four films using an IR microscope. Presence of TM across the film was monitored using density of the principal peaks of TM which were previously identified and confirmed by ATR-FTIR results i.e. 2976, 2892 and $1698 \mathrm{~cm}^{-1}$. Figure 6 illustrates the IR mapping results using F1 as a representative formulation which includes the 3D map of the film (top), the density map of absorption (middle) and the evaluated IR peaks (bottom). The results suggest 40-60\% (green) presence of TM across the film (3D map) with minor areas containing less than $30 \%$ (amber and red) present. Occasional appearance of blue spots in the map indicates areas with TM density of above $70 \%$ but these only appeared in a

601

602

603

604

605

606

607

608

609

610

611

612

613

614

615

616

617

618

619

620

621

622

623

few instances. This indicates higher density of drug particles in those areas compared to areas appearing in green. Formulations F2-F4 also showed similar results to F1 with adequate uniform distribution of TM across the film. An ideal IR map must show even density (40-60\%) of drug molecules across the film indicated by green color for principal peaks. For improved distribution, the time for gelation and mixing process could be extended with higher stirring speed to ensure the drug molecules are more evenly distributed.

\subsection{Thermogravimetric analysis (TGA)}

Residual moisture content of the films was determined by TGA. Formulation F1 with $8.68 \%$ and F2 with 5.96\% showed the highest and lowest moisture content, respectively, amongst all films. Despite the effect of GLY on moisture content which was reported by Ahmed and Boateng (2018), the structure of each polymer and their ability to absorb water play an important part in moisture content of the prepared formulations. F1 containing only HA, had the highest $\%$ moisture content which again confirms the exceptional hydration characteristic of this polymer, as was observed during swelling studies (figure 3) due to its hydrophilic nature. The ability of HA to absorb water molecules is due to presence of amine group (not present in HPMC) which provides additional intermolecular polymer-polymer interaction, allowing the water molecules to remain between the polymer chains without collapse of the film matrix structure. Percentage moisture content in F3 and F4 evidently support this characteristic of HA. F3 containing 1:1 ratio of HPMC:HA showed higher \% moisture content, (7.54\%), compared to F4, $(6.72 \%)$. The moisture content of $7.54 \%$ in formulation F3 was also higher than that in formulation F2 (5.96\%) which contained only HPMC, again demonstrating the impact of HA in the films holding on to more moisture compared to HPMC. Furthermore, F4 containing higher ratio of HPMC with ratio of HPMC:HA 3:1, showed lower \% moisture content 
compared to F3 but higher value compared to F2 which again support the higher moisture holding capacity of HA.

\subsection{Modulated differential scanning calorimetry (MDSC)}

DSC analysis was used to characterize the thermal behavior of pure TM and its physical state when incorporated within formulations F1-F4. HPMC and HA are both predominantly amorphous polymers and typically expected to exhibit a phase transition at specific temperature threshold known as glass transition temperature $\left(\mathrm{T}_{\mathrm{g}}\right)$. Absence of $\mathrm{T}_{\mathrm{g}}$ was expected for HA as previous DSC analysis of this polymer also showed no clear $\mathrm{T}_{\mathrm{g}}$ (Ravari et al, 2016; Abdelkader et al., 2016). Jadhav and colleagues reported $\mathrm{T}_{\mathrm{g}}$ of HPMC at $180{ }^{\circ} \mathrm{C}$. However, the DSC thermogram of HPMC powder used in this study showed $\mathrm{T}_{\mathrm{g}}$ between $125-137^{\circ} \mathrm{C}$ followed by distinct endothermic thermal event at $164.21{ }^{\circ} \mathrm{C}$. This is fundamentally based on different grades of this polymer (i.e. molecular weight) [the HPMC grade was not specified in the abovementioned study]. Furthermore, no clear $\mathrm{T}_{\mathrm{g}}$ was observed in the thermograms of the films (figure 7b). The exothermic peak in F1 is typical for HA, normally observed between 200-230 ${ }^{\circ} \mathrm{C}$ and corresponds to crystallization of HA. This peak was observed in the thermogram of pure polymer powder, as a shoulder to the large endothermic peak at $205.77{ }^{\circ} \mathrm{C}$ (likely due to trace components such as sulfate linked with glycosaminoglycan), but shifted to around 160 ${ }^{\circ} \mathrm{C}$ (figure $7 \mathrm{~b}$ ) in the HA only film (F1) as the polymer undergoes further amorphization during film formation due to presence of GLY and residual water content (Adel and ElKasabgy, 2014). DSC thermogram of pure TM displayed typical thermogram of a crystalline substance with a single sharp endothermic peak at $205.11^{\circ} \mathrm{C}$ corresponding to its melting point (figure $7 \mathrm{a}$ ). The sharp characteristic peak of TM was absent in DSC thermograms of formulations F1-F4 indicating the suppression of TM crystallinity in the films. This amorphization of the drug also suggests distribution and molecular dispersion of TM within the film matrices. In general, amorphous drugs exhibit better solubility and therefore more rapid release advantage over the more stable crystalline equivalent, however, they have the tendency to convert back to the stable crystalline form and must therefore be stored appropriately.

\subsection{Scanning electron microscopy (SEM)}

The SEM micrographs of all the films revealed general smoothness of the surface as demonstrated in figure 8 . Considering the high sensitivity of the eye, it is crucial that the ocular films aimed for topical administration are completely smooth and causes no irritation for the patient. Occasionally, small particles appeared sparsely on the surface of some films in various 
batches of different formulations; for instance, single small particle could be observed on the surface of F3 (figure 8). After chemical analysis of these particles by semi-quantitative EDX at $8 \mathrm{kV}$ accelerating voltage, they appear to be mainly entangled polymers identified by high carbon $(64.9 \pm 0.7 \%)$ and oxygen content $(34.1 \pm 2.1 \%)$ in their molecular structure. Negligible amount of aluminium $(1.0 \pm 0.3 \%)$ was detected by EDX which is due to X-ray going through the thin and transparent film samples and reaching the aluminium stub.

\subsection{XRD}

The diffraction patterns of TM powder revealed numerous sharp and high intensity peaks between diffraction angles range of $10^{\circ}$ to $30^{\circ} 2 \theta$, confirming the crystallinity of TM powder a shown in the DSC results. HPMC and HA powders exhibited halo diffraction patterns which confirm their amorphous nature. Generally, the diffractograms of formulations F1-F4 showed halo diffraction pattern which indicates amorphization of the drug and its molecular distribution in the film matrices. This supports DSC and IR microscopy results confirming the molecular dispersion of TM throughout the films. A sharp peak appeared initially in the diffractograms of F1-F4 at $23^{\circ} 2 \theta$ similar to HPMC containing formulations reported by of Okeke and Boateng (2016). After further evaluation of various formulations including blank and unplasticised films, the peak was determined to be a response to the plastic sample holder of the specimen during sample analysis. After removing the plastic part of the specimen holder the peak disappeared and the expected halo diffraction pattern of amorphous film was observed (figure 9).

\subsection{In vitro drug release}

In vitro - in vivo correlation (IVIVC) of drug release, permeation and efficacy of TM at known concentrations has been investigated by many scientists including Shafie and Rady (2012), Korogiannaki et al (2015), Thakral et al (2015) and Desai et al (2018). TM has been the first line treatment for glaucoma for many years and there are many studies available which have used TM as the model drug, since its therapeutic efficacy is well-known. However, the challenges associated with more effective delivery of TM still exist as eye drops (ophthalmic solutions) are still the only dosage form available for topical delivery of TM. Conventionally, maximum TM dosage is one drop of $0.5 \% \mathrm{w} / \mathrm{v}$ applied in the affected eye twice daily (total dose of $400 \mu \mathrm{g}$ in $24 \mathrm{hrs}$ ) which provides plasma concentrations of approximately $0.5 \mathrm{ng} \mathrm{ml}^{-1}$, based on a study which used healthy volunteers (Gray, 2006). More importantly dosages above 
one drop of $0.5 \% \mathrm{w} / \mathrm{v}$ TM ophthalmic solution twice a day generally have not been shown to produce further reduction in IOP (Bauch \& Lomb Timoptic $\left.{ }^{\circledR}\right)$.

In this study, $0.5 \% \mathrm{w} / \mathrm{v}$ dose of TM was incorporated into the gels prior to film formation volume. The film disks used for in vitro drug release studies contained the following total available dose for formulations F1 to F4, respectively: $196 \mu \mathrm{g}, 160 \mu \mathrm{g}, 129 \mu \mathrm{g}$ and 227 $\mu \mathrm{g}$. Figure 10 shows the in vitro cumulative release profiles of the TM loaded ocular films F1F4 using an automated flow cell. The flow rate $\left(50 \mu \mathrm{L} \mathrm{min}^{-1}\right)$ was the lowest on the machine and closest to the tear turnover rate in human eyes $\left(0.5-2.2 \mu \mathrm{L} \mathrm{min}{ }^{-1}\right)$ or total tear volume in a healthy eye $(7-9 \mu \mathrm{L})$ (Bachu et al., 2018) which could produce sufficient volume of dissolution medium for in vitro analysis of drug release. F1 showed highest cumulative release of $71.59 \%(140.32 \mu \mathrm{g})$ whilst F4 showed the lowest with $41.48 \%$ (94.16 $\mu \mathrm{g})$ release. The films containing HA i.e. F1, F3 and F4 reached maximum cumulative drug release in 8 hrs which shows remarkable ability of HA to delay the polymer chain disentanglement and therefore slows down rate of drug diffusion from the swollen matrix resulting in a prolonged release pattern of the drug from these formulations. However, F2 reached maximum cumulative drug release within only $2 \mathrm{hrs}$, which is due to rapid swelling and erosion rate. This result is in agreement with the swelling index study where F1, F3 and F4 (in order) showed prolonged swelling and delayed erosion compared with F2 which swelled more rapidly and eventually disintegrated.

Calles and colleagues investigated TM release from cross-linked ocular films and 80\% TM was released in the first $2 \mathrm{hrs}$ of study (Calles et al., 2013). In another study by Mehta and co, TM was used in polymeric contact lens coating, and 75\% TM was released within 6 hrs (Mehta et al., 2017). The ability of HA to delay the release of TM in F1, F3 and F4 of this study shows potential of this polymer for controlled ocular drug delivery purposes in the form of thin films. The flow rate of STF over the samples in this study $\left(50 \mu \mathrm{L} \mathrm{min}{ }^{-1}\right)$ is considerably higher than usual tear turnover rate in the eye $\left(1.2 \mu \mathrm{L} \mathrm{min}^{-1}\right)$ or total tear volume in the ocular cavity $(7-10$ $\mu \mathrm{L})$; therefore, the release of drug from the formulations in this study is expected to be even more prolonged in an in vivo setting. This was also confirmed by the in vivo study carried out by Desai and colleagues. In this study, the poor IVIVC observed was due to the differences between the in vivo and in vitro release conditions and more specifically the dissolution volume under sink conditions. In their in vitro study, the formulation was placed in $2 \mathrm{~mL}$ of STF dissolution media, whereas in the animal study, the formulation was exposed to just $7-10 \mu \mathrm{L}$ of the tear volume in the ocular cavity. A markedly high release of TM was observed under the in vitro release conditions, whereas a lower (2-fold) release amount was observed in the rabbit 
tear fluid (Desai et al., 2018). Therefore, the release of TM from erodible thin ocular films in this study is also expected to be more prolonged during the in vivo studies where the tear volume and turnover rate is significantly lower. Therefore, despite the low thickness, the HAcontaining formulations developed in this study, have the ability to deliver up to $71 \%$ of the dose $(\approx 140.32 \mu \mathrm{g})$ in a controlled release pattern, within 8 hours. However, the concentration of the drug in each formulation needs to be increased to reach the required $400 \mu \mathrm{g}$ dose a day before proceeding to in vivo testing of these ocular films in future work.

Further, mechanism and kinetics of drug release from the films were calculated according to Korsmeyer-Peppas model, which describes the release behavior of drug from polymeric systems which is related to the erosion and dissolution of the polymer matrix (Korsmeyer et al., 1983).

$\frac{Q_{t}}{Q_{\infty}}=k t^{n}$

Where $\mathrm{Q}_{t} / \mathrm{Q}_{\infty}$ is the cumulative percent release, $\mathrm{k}$ is Korsmeyer-Peppas constant, $\mathrm{t}$ is the release time and $\mathrm{n}$ the release exponent for the drug. Korsmeyer-Peppas stated that the above equation could adequately describe the release of solutes from slabs, spheres, cylinders and discs, regardless of the release mechanism.

The value of ' $n$ ' gives an indication of the release mechanism; when $n=1$, the release rate is independent of time (zero-order) (case II transport), $\mathrm{n}=0.5$ represents Fickian diffusion, $\mathrm{n}<$ 0.5 indicates that the release rates exhibit a combined mechanism of diffusion partially through a swollen matrix and partially through water-filled pores and when $0.5<\mathrm{n}<1.0$, diffusion and non-Fickian transport are implied, while $\mathrm{n}$ values $>1.0$ implies super case II transport. The release exponent, $\mathrm{n}$, is the slope value of $\log \mathrm{Q}_{\mathrm{t}} / \mathrm{Q}_{\infty}$ versus $\log$ time curve. Slope values presented in table 5 showed values of $0.5<\mathrm{n}<1.0$ for F1 to F4 which suggests that the release of TM from all formulations followed non-Fickian diffusion mechanism.

\subsection{Cytotoxicity and cell viability}

Assessment of cytotoxicity is vital for any materials that come into contact with the ocular surface. Formulations F1-F4 were applied to HeLa cell lines for cytotoxicity evaluation of the films. The polymers used in this study are currently being used in many pharmaceutical formulations and are listed as GRAS by FDA. The MTT assay in this study investigated blank single polymer HA (F1) and HPMC (F2) film as well as F3 and blank F3. The results (figure 11) revealed high \% cell viability of polymers and the TM loaded films over $72 \mathrm{hrs}$. Generally, 
the accepted $\%$ cell viability is expected to be $>70 \%$ according to the ISO specification (Ahmed et al., 2018) and all the formulations tested (both blank and TM loaded) show cell viability values above $70 \%$ which confirm their suitability for direct application to the ocular surface for up to $72 \mathrm{hrs}$.

\subsection{Conclusion}

The results obtained in this study reveal that HA and HPMC can produce optimum ocular films either as single polymer or in composite matrix. However, incorporating both polymers within a composite formulation can combine the strong film forming ability of HPMC with the remarkable swelling capacity of the HA. The strong amino and hydroxyl group in HA was shown to play a major role in its ability to absorb and retain water molecules for longer period, which allows prolonged drug release profiles. The drug loaded films were generally biocompatible with cell viability results falling within the expected standard. Overall, composition of HA and HPMC have enhanced characteristics compared to single polymer formulations and is a promising delivery system for topical delivery of TM for potential treatment and management of glaucoma, and this will be further evaluated using an in vivo animal study in future work.

\section{References}

Abdelkader, H., Longman, M.R., Alany, R.G., Pierscionek, B. 2016. Phytosome-hyaluronic acid systems for ocular delivery of L-carnosine. Int. J. Nanomed. 11(1), 2815-2827.

Adel S. and ElKasabgy N.A. 2014. Design of innovated lipid-based floating beads loaded with an antispasmodic drug: in-vitro and in-vivo evaluation. J. Lipos. Res. 24 (2), 136-149.

Ahmed, A., Boateng J. 2018. Calcium alginate-based antimicrobial film dressings for potential healing of infected foot ulcers. Ther. Del. 9(3), 185-204.

Ahmed, A., Getti, G., Boateng, J.S. 2018. Ciprofloxacin-loaded calcium alginate wafers prepared by freeze-drying technique for potential healing of chronic diabetic foot ulcers. Drug Del. Transl. Res. 8(6), 1751-1768.

Alariqi, S.AS., Mutair, A.A., Singh R.P. 2016. Effect of Different Sterilization Methods on Biodegradation of Biomedical Polypropylene. J. Environ. Anal. Toxicol. 6(373), 1-7.

Aulton, M.E., Taylor K.M.G. 2017. Pharmaceutics: Science of Dosage Form Design. 5th ed. UK: Elsevier. pp 710-732. 
790

791

792

793

794

795

796

797

798

799

800

801

802

803

804

805

806

807

808

809

810

811

812

813

814

815

816

817

818

Ayensu, I., Mitchell, J.C., Boateng J.S. 2012. Effect of membrane dialysis on characteristics of lyophilized chitosan wafers for potential buccal delivery of proteins. Int. J. Biol. Macromol. 50, 905-909.

Bachu R.D., Chowdhury P., Al-Saedi Z.H.F., Karla P.K. and Boddu S.H.S. 2018. Ocular Drug Delivery Barriers - Role of Nanocarriers in the Treatment of Anterior Segment Ocular Diseases. Pharm. 10 (28), 1-31.

Bhamra, T.S., Tighe, B.J. 2017. Mechanical properties of contact lenses: The contribution of measurement techniques and clinical feedback to 50 years of materials development. Contact Lens Ant. Eye. 40(2), 70-81.

Boateng J.S., Popescu A.M. 2016. Composite bi-layered erodible films for potential ocular drug delivery. Coll. Surf. B: Biointerf. 145(2), 353-361.

Brannigan, R.P., Khutoryanskiy, V.V. 2017. Synthesis and evaluation of mucoadhesive acryloyl-quaternized PDMAEMA nanogels for ocular drug delivery. Coll. Surf. B: Biointerf. 155(1), 538-543.

Calles, J.A., Tártara, L.I., Lopez-García, A., Diebold, Y., Palma, S.D., Vallés, E.M. 2013. Novel bioadhesive hyaluronan-itaconic acid crosslinked films for ocular therapy. Int. J. Pharm. $455,48-56$.

Chavda, D., Thakkar, V., Soni, T., Gandhi, T. 2016. Formulation and in vitro - in vivo evaluations of Timolol Maleate viscous eye drops for the treatment of glaucoma. Eur. J. Biomed. Pharm. Sci. 3(9), 573-585.

Desai A.R., Maulvi F.A., Pandya M.M., Ranch K.M., Vyas B.A., Shah S.A., Shah D.O. 2018. Co-delivery of timolol and hyaluronic acid from semi-circular ring-implanted contact lenses for the treatment of glaucoma: in vitro and in vivo evaluation. Biomater. Sci. 6, 1580-1591.

ElShaer, A., Mustafa, S., Kasar, M., Thapa, S., Ghatora, B., Alany, R.G. 2016. Nanoparticleladen contact lens for controlled ocular delivery of prednisolone: formulation optimization using statistical experimental design. Pharm. MDPI. 8(14), 1-16.

Fuentes, R., Fernandez, E., Pascual, I., Garcia, C. 2013. UV-Visible Transmittance of SiliconeHydrogel Contact Lenses measured with a fiber optic spectrometer. Int. Soc Optics Photon. Tech. 8785(8785AZ), 1-9. 
819 Galante, R., Oliveira, A.S., Topete, A., Ghisleni, D., Braga, M., Pinto, T.J.A., Colac R., Serro, 820 A.P. 2018. Drug-eluting silicone hydrogel for therapeutic contact lenses: Impact of sterilization methods on the system performance. Coll. Surf. B: Biointerf. 161, 537-546.

822 Gray C. 2006. Cardiac and central nervous system toxicity with timolol eye drops in an infant: 823 case report and discussion. Pediatr. Perinat. Drug Ther. 7 (1), 15-18.

824 Gulsen D. and Chauhan A. 2004. Ophthalmic Drug Delivery through Contact Lenses. Investig. Ophthalmol. Visual Sci. 45 (7), 2342-2347.

826 Hashemi, H., Khabazkhoob, M., Saatchi, M., Ostadimoghaddam, H., Yekta A. 2017. Visual 827 impairment and blindness in a population-based study of Mashhad, Iran J. Curr. Ophthalmol. 828 $1-8$.

829

Hui, A. 2017. Contact lenses for ophthalmic drug delivery. Clin. Experiment. Optom. 100, 830 $494-512$.

Imperiale, J.C., Acosta, G.B., Sosnik, A. 2018. Polymer-based carriers for ophthalmic drug delivery. J. Contr. Rel. 285, 106-141. Jethava, J.K., Jethava, G.K. 2014. Design, formulation, and evaluation of novel sustain release bioadhesive in-situ gelling ocular inserts of ketorolac tromethamine. Int. J. Pharm. Investig. 4 (4), 226-232.

Jin Q., Li H., Jin Z., Huang L., Wang F., Zhou Y., Liu Y., Jiang C., Oswald J., Wu J. and Song 837 X. 2018. TPGS modified nanoliposomes as an effective ocular delivery system to treat glaucoma. Int. J. Pharm. 553, 21-28.

839 Jons, J.B., Aung, T., Bourne, R.R., Bron A.M., Ritch, R., Panda-Jonas, S. 2017. Glauc. Sem. $840 \quad 3(12), 1-11$.

841 Karki, S., Kim, H., Na, S.J., Shin, D., Jo, K., Lee, J. 2016. Thin films as an emerging platform 842 for drug delivery. Asian J. Pharm. Sci. 2, 559-574.

843 Khan, S., Trivedi, V., Boateng, J.S. 2016. Functional physico-chemical, ex vivo permeation 844 and cell viability characterization of omeprazole loaded buccal films for pediatric drug 845 delivery. Int. J. Pharm. 500, 217-226. 
846 Korogiannak M., Guidi G., Jones L. and Sheardown H. 2015. Timolol maleate release from 847 hyaluronic acid-containing model silicone hydrogel contact lens materials. Biomater.Appl. 30 848 (3), 361-376.

849 Korsmeyer R.W., Gurny R., Doelker E., Buri P. and Peppas N.A. 1983. Mechanisms of Solute 850 Release from Porous Hydrophilic Polymers. Int. J. Pharm. 15 (1), 25-35.

851 Mandal, A., Bisht, R., Rupenthal, I.D., Mitra, A.K. 2017. Polymeric micelles for ocular drug 852 delivery: From structural frameworks to recent preclinical studies. J. Contr. Rel. 248, 96-116. Mehta, P., Al-Kinani, A.A., Arshad, M.S., Chang, M.W., Alany R.G., Ahmad, Z. 2017. Development and characterization of electrospun timolol maleate-loaded polymeric contact lens coatings containing various permeation enhancers. Int. J. Pharm. 532, 408-420. Momoh, F.U., Boateng, J.S., Richardson, S.C.W., Chowdhry, B.Z., Mitchell, J.C. 2015. Development and functional characterization of alginate dressing as potential protein delivery system for wound healing. Int. J. Biol. Macromol. 81(3), 137-150.

Morrison P.W.J and Khutoryanskiy V.V. 2014. Advances in ophthalmic drug delivery. Ther. Del. 5 (12), 1297-1315.

Morrison, P.W.J., Khutoryanskiy, V.V. 2014. Enhancement in Corneal Permeability of Riboflavin Using Calcium Sequestering Compounds. Int. J. Pharm. 472, 56-64.

Nowak, J., Laffleur, F., Bernkop-Schnürch, A. 2015. Preactivated hyaluronic acid: A potential 864 mucoadhesive polymer for vaginal delivery. Int. J. Pharm. 478, 383-389.

Okeke, O.C., Boateng, J.S., 2016. Composite HPMC and sodium alginate based buccal formulations for nicotine replacement therapy. Int. J. Biol. Macromol. 91, 31-44.

Papakonstantinou, E., Roth, M., Karakiulakis, G. 2012. Hyaluronic acid: A key molecule in 868 skin aging. Dermatoendocrinol. 4(3), 253-258.

Phadtare, D., Phadtare, G., Nilesh, B., Asawat, M. 2014. Hypromellose - A choice of polymer in extended release tablet formulation. World J. Pharm. Pharm. Sci. 3(9), 551-565.

871 Priya, K.R.N, Bhattacharyya, S., Ramesh, B.P. 2014. Formulation and evaluation of erodible ocular films of valacyclovir hydrochloride. Dhaka Univ. J. Pharm. Sci. 13(1), 75-81. Human Tear Fluid Lipidome: From Composition to Function. PLOS ONE. 6 (5), 1-7. 
875 Rastogi, R.P., Richa, Kumar A., Tyagi, M.B., Sinha, R.P. 2010. Molecular mechanisms of 876 ultraviolet radiation-induced DNA damage and repair. J. Nucleic Acids. 16, 1-32.

877 Ravari, N.S., Goodarzi, N., Alvandifar, F., Amini, M., Souri, E., Khoshayand, M.R., Mirzaie, 878 Z.H., Atyabi, F., Dinarvand, R. 2016. Fabrication and biological evaluation of chitosan coated 879 hyaluronic acid-docetaxel conjugate nanoparticles in CD44+ cancer cells. Daru J. Pharm. Sci. $88024(21), 1-12$.

881 Rodriguez, I., Vazquez, J.A., Pastrana, L., Khutoryanskiy, V.V. 2017. Enhancement and 882 inhibition effects on the corneal permeability of Timolol Maleate: Polymers, Cyclodextrins and 883 Chelating Agents. Int. J. Pharm. 529, 168-177.

Shafie M.A.A. and Rady M.A.H. 2012. In vitro and In vivo Evaluation of Timolol Maleate 885 Ocular Inserts Using Different Polymers. J. Clin. Exp. Ophthalmol. 3 (8), 1-9.

Stokroos I., Kalicharan D., Van Der Want J.J.L. and Jongebloed W.L. 1997. A comparative 887 study of thin coatings of $\mathrm{Au} / \mathrm{Pd}, \mathrm{Pt}$ and $\mathrm{Cr}$ produced by magnetron sputtering for FE-SEM. J. Microscop. 189 (1), 79-89.

889

Thakral S., Issarani R. and Nagori B.P. 2015. Formulation and In Vitro-In Vivo Correlation of 890 Timolol Maleate Ocular Insert. Indones. J. Clin. Pharm. 4 (4), 281-288.

891 Tranoudis, I., Efron, N. 2004. Tensile properties of soft contact lens materials. Contact Lens 892 Anter. Eye. 27, 177-191. Wang F., Bao X., Fang A., Li H., Zhou Y., Liu Y., Jiang C., Wu J. and Song X. 2018. 894 Nanoliposome-Encapsulated Brinzolamide-hydropropyl- b-cyclodextrin Inclusion Complex: A Potential Therapeutic Ocular Drug-Delivery System. Front. Pharmacol. 9 (91), 1-9. 1717.

898 Yeh, C.C., Chen, C.N., Li, Y.T., Chang, C.W., Cheng, M.Y., Chang, H.I. 2011. The effect of 899 polymer molecular weight and UV radiation on physical properties and bioactivities of PCL 900 films. Cell. Polym. 30(5), 261-275.

901 Zafar, A., Ahmad, J., Akhter, S., Addo, R.T. 2016. Nanotechnology for transcorneal drug 902 targeting in glaucoma: challenges and progress. In: Addo R. (Ed) Ocular drug delivery: 903 advances, challenges and applications ( $1^{\text {st }}$ Edition). Springer, Cham, 75-99. 
905

906 Table 1. Formulation (gel) composition of each film in $100 \mathrm{~mL}$ of water. All gel 907 formulations were loaded with $0.5 \% \mathrm{w} / \mathrm{v}$ of TM prior to drying in oven.

\section{Composition in $100 \mathrm{~mL}$ twice-distilled water}

\begin{tabular}{lllll} 
Formulation & HPMC (mg) & HA $(\mathbf{m g})$ & GLY (mg) & TM (mg) \\
\hline F1 & - & 1000 & 500 & 7.50 \\
F2 & 1500 & - & 750 & 11.25 \\
F3 & 500 & 500 & 500 & 7.50 \\
F4 & 500 & 500 & 1000 & 15.00 \\
\hline
\end{tabular}

908

909

Table 2. Weight, thickness, surface pH and folding endurance results of formulations F1910 F4 $(n=3)$.

\begin{tabular}{ccccc}
\hline $\begin{array}{c}\text { TIM-loaded } \\
\text { Film }\end{array}$ & $\begin{array}{c}\text { Weight }(\mathbf{g}) \\
( \pm \text { SD })\end{array}$ & $\begin{array}{c}\text { Thickness }(\mathbf{m m}) \\
( \pm \text { SD })\end{array}$ & $\begin{array}{c}\text { Surface } \mathbf{p H} \\
( \pm \mathbf{S D})\end{array}$ & $\begin{array}{c}\text { Folding Endurance } \\
( \pm \mathbf{S D})\end{array}$ \\
\hline F1 & $0.10 \pm 0.01$ & $0.04 \pm 0.01$ & $5.97 \pm 0.08$ & $>300$ \\
F2 & $0.12 \pm 0.02$ & $0.07 \pm 0.03$ & $6.46 \pm 0.05$ & $>300$ \\
F3 & $0.09 \pm 0.01$ & $0.06 \pm 0.01$ & $6.05 \pm 0.07$ & $>300$ \\
F4 & $0.17 \pm 0.03$ & $0.09 \pm 0.01$ & $6.01 \pm 0.03$ & $>300$
\end{tabular}


913 Table 3. Tensile properties of formulations F1-F4 $(n=3)$.

\begin{tabular}{cccc}
\hline $\begin{array}{c}\text { TIM-loaded } \\
\text { Film }\end{array}$ & $\begin{array}{c}\text { Tensile Strength }\left(\mathbf{N} / \mathbf{m m}^{\mathbf{2}}\right) \\
\pm \text { SD }\end{array}$ & $\begin{array}{c}\text { Elastic Modulus (mPa) } \\
\pm \text { SD }\end{array}$ & $\begin{array}{c}\text { Elongation (\%) } \\
\pm \text { SD }\end{array}$ \\
\hline F1 & $21.01 \pm 4.64$ & $0.98 \pm 0.25$ & $72.53 \pm 6.37$ \\
F2 & $198.97 \pm 17.31$ & $4.80 \pm 1.23$ & $58.01 \pm 9.12$ \\
F3 & $49.72 \pm 5.42$ & $2.38 \pm 0.23$ & $51.66 \pm 0.81$ \\
F4 & $149.07 \pm 21.93$ & $5.38 \pm 0.69$ & $51.19 \pm 12.33$
\end{tabular}

914

915

916

Table 4. Mucoadhesion properties of formulations F1-F4 $(n=3)$.

\begin{tabular}{cccc}
\hline TIM-loaded Film & PAF (N) & TWA (N/s) & Cohesiveness (mm) \\
\hline F1 & $1.66 \pm 0.42$ & $1.76 \pm 0.54$ & $6.49 \pm 1.13$ \\
F2 & $0.98 \pm 0.06$ & $0.58 \pm 0.05$ & $1.79 \pm 0.33$ \\
F3 & $3.79 \pm 0.43$ & $5.85 \pm 0.53$ & $5.22 \pm 0.36$ \\
F4 & $3.47 \pm 0.61$ & $3.84 \pm 0.55$ & $5.38 \pm 0.50$
\end{tabular}

917

918

919 Table 5. In vitro slopes and regression values from the Korsmeyer-Peppas kinetic model.

\begin{tabular}{lll}
\hline TM-loaded Film & $\mathbf{R}^{\mathbf{2}}$ & $\mathbf{n}$ \\
\hline F1 & 0.996 & 0.797 \\
F2 & 0.996 & 0.683 \\
F3 & 0.996 & 0.853 \\
F4 & 0.997 & 0.898 \\
\hline
\end{tabular}



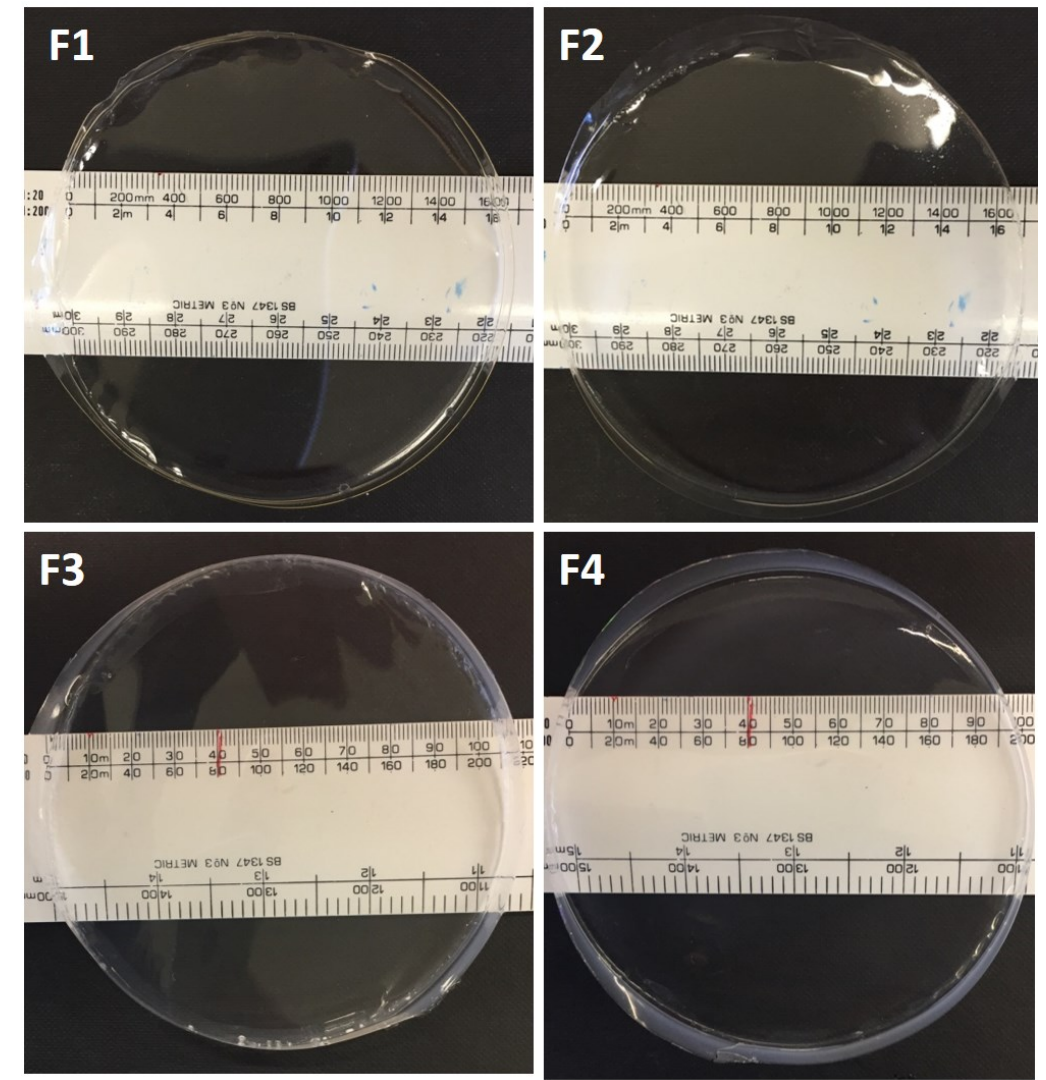

Figure 1. Digital images of the ocular films (F1-F4) against a numbered ruler as part of visual assessment of transparency.

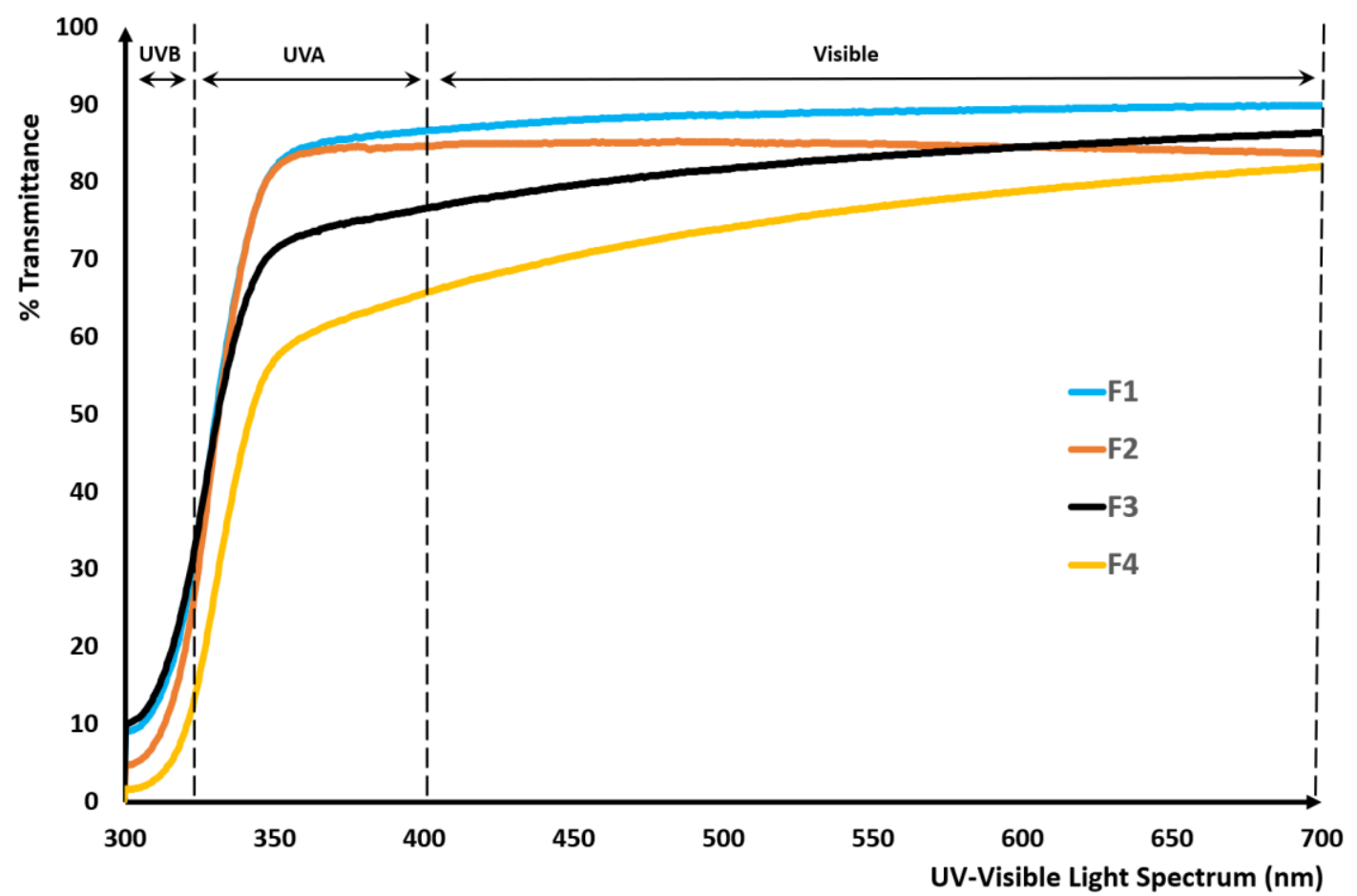

Figure 2. Transmittance spectra (ultraviolet (UV)-visible range) for the tested formulations F1-F4. 


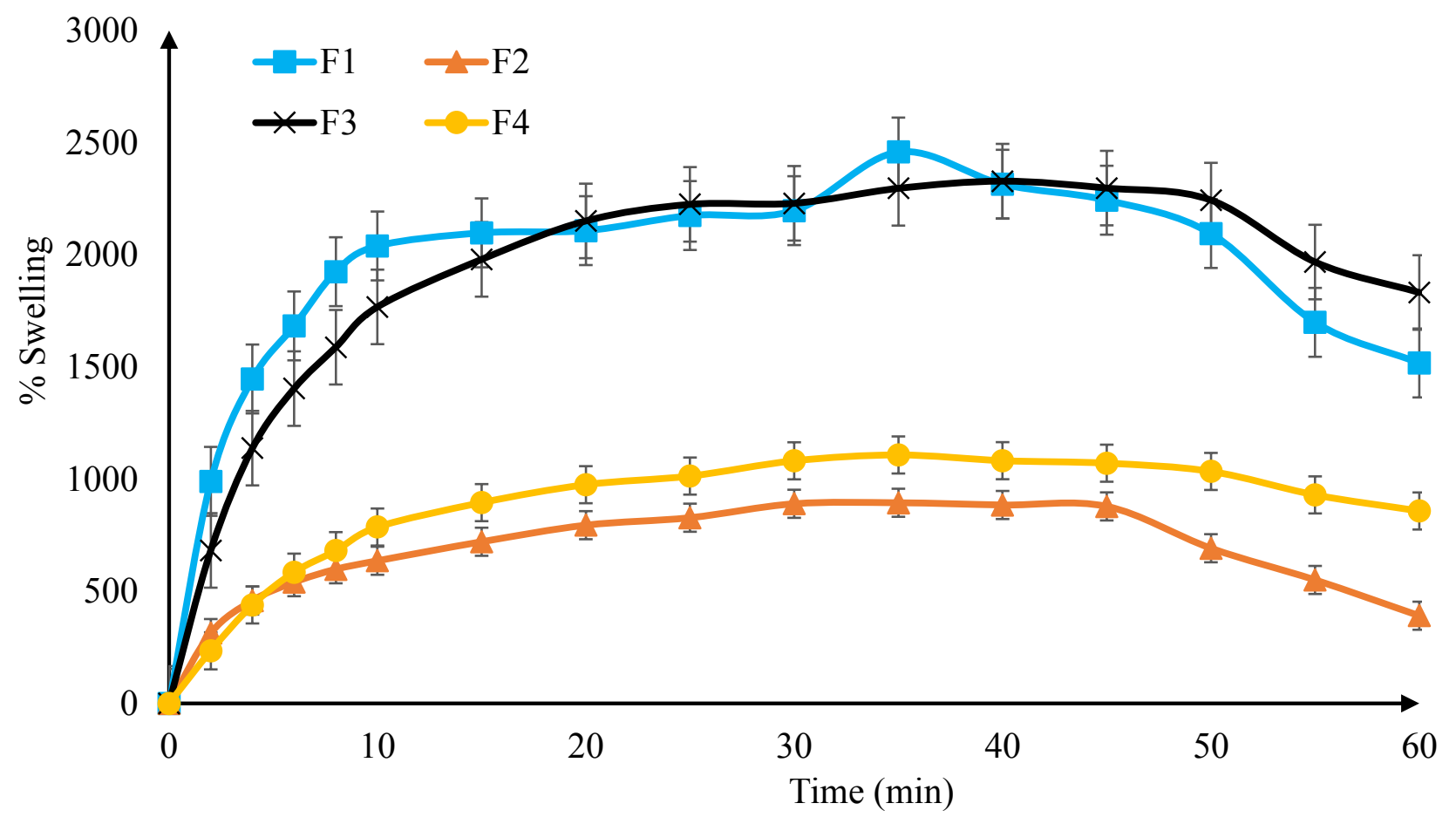

930

931

932

933

934

935

936

Figure 3. Swelling profiles of the formulations F1-F4 (mean $\pm \mathrm{SD}, n=3)$.

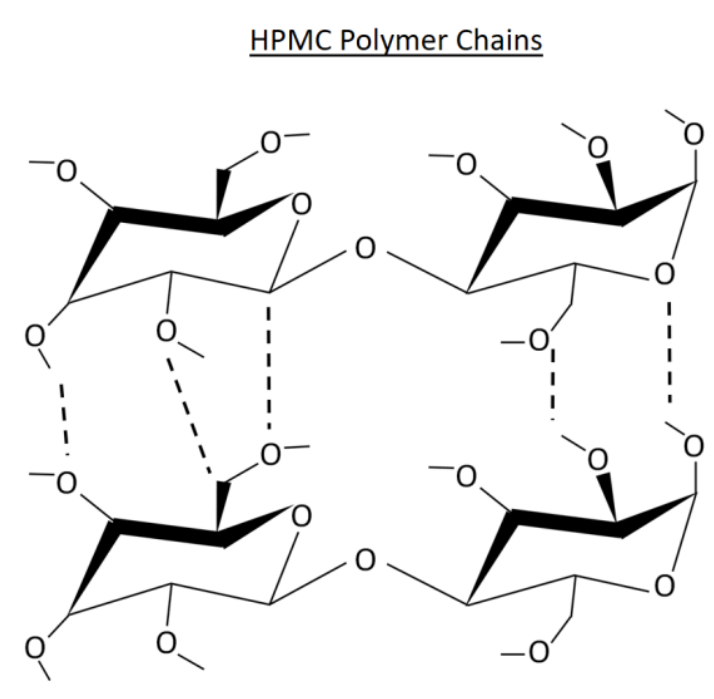

Figure 4. Molecular interaction schematic of HPMC (left) and HA (right) polymer chains illustrating additional hydrogen bond in HA matrix due to presence of nitrogen (as indicated in red). 


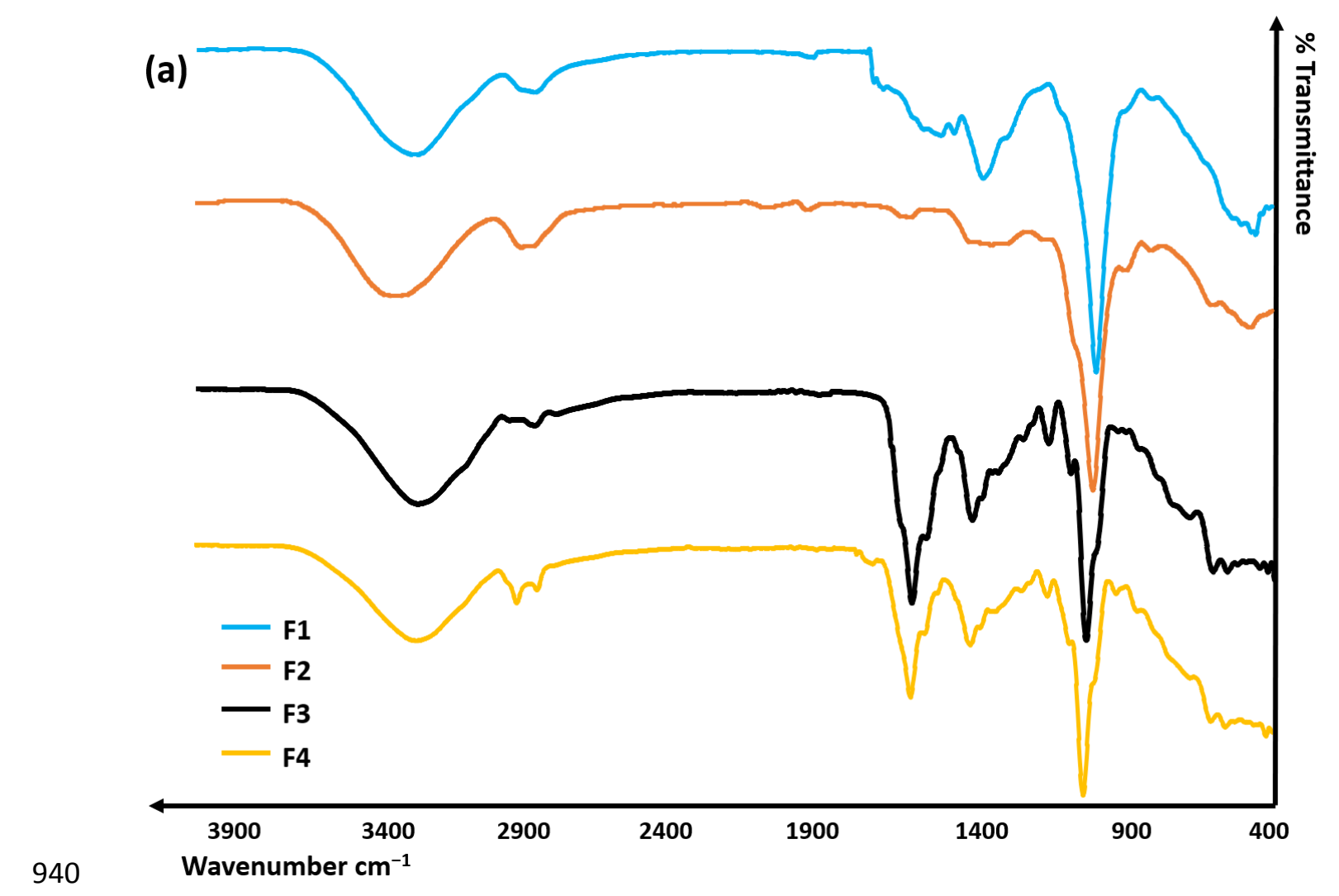

941

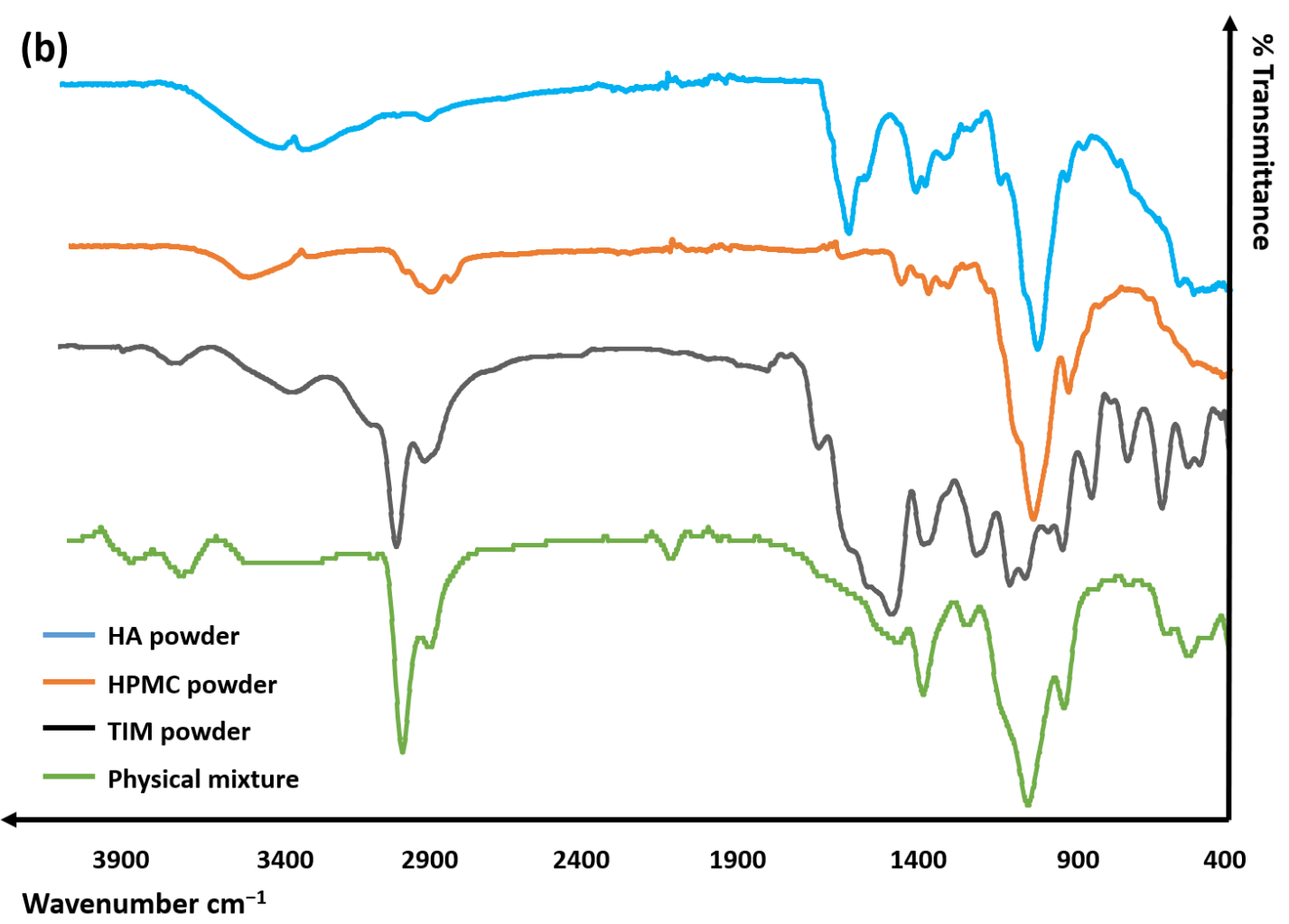

Wavenumber $\mathrm{cm}^{-1}$

Figure 5. FTIR spectra of the (a) films and (b) TM, polymer powders and physical mixture. 


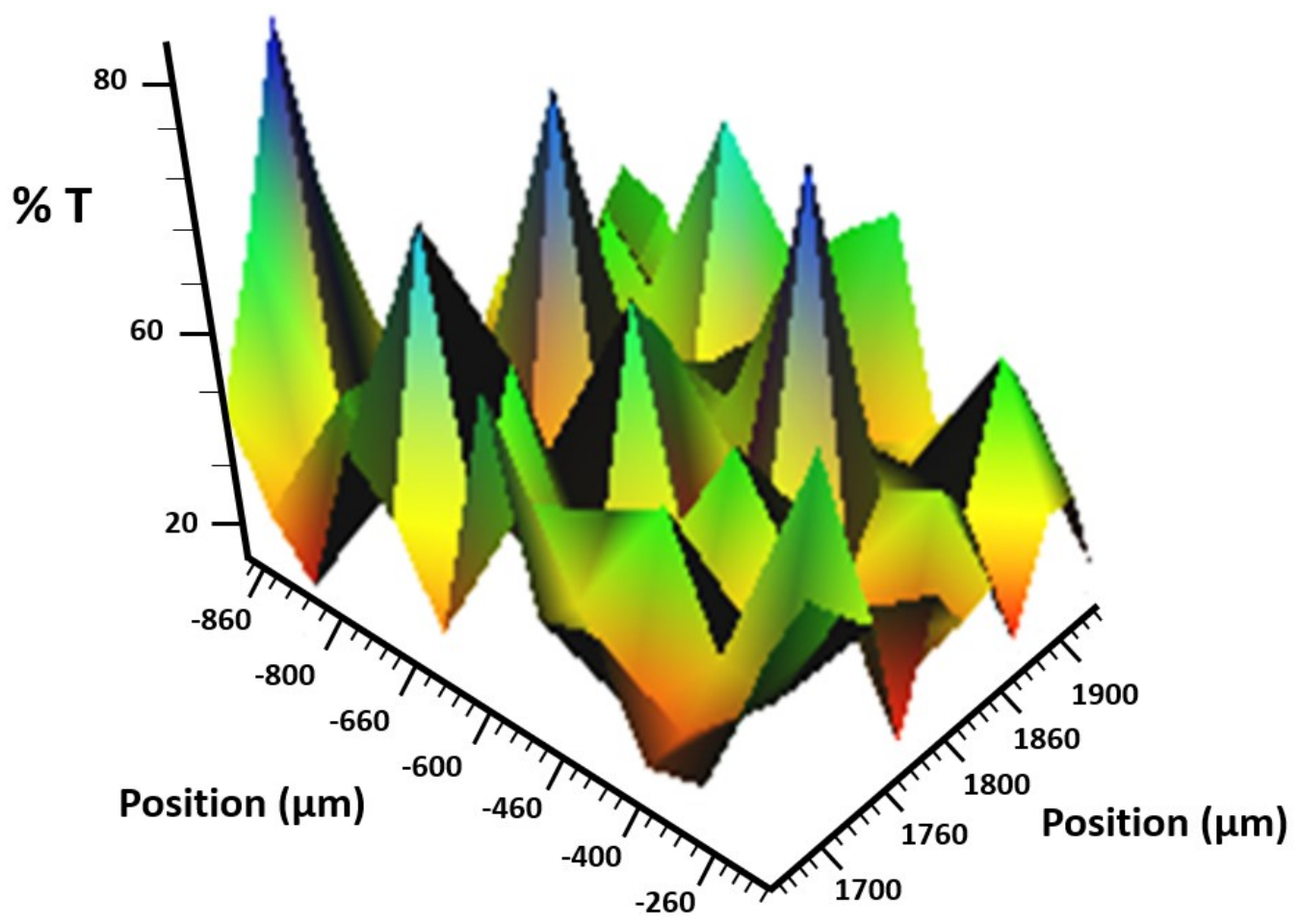

947

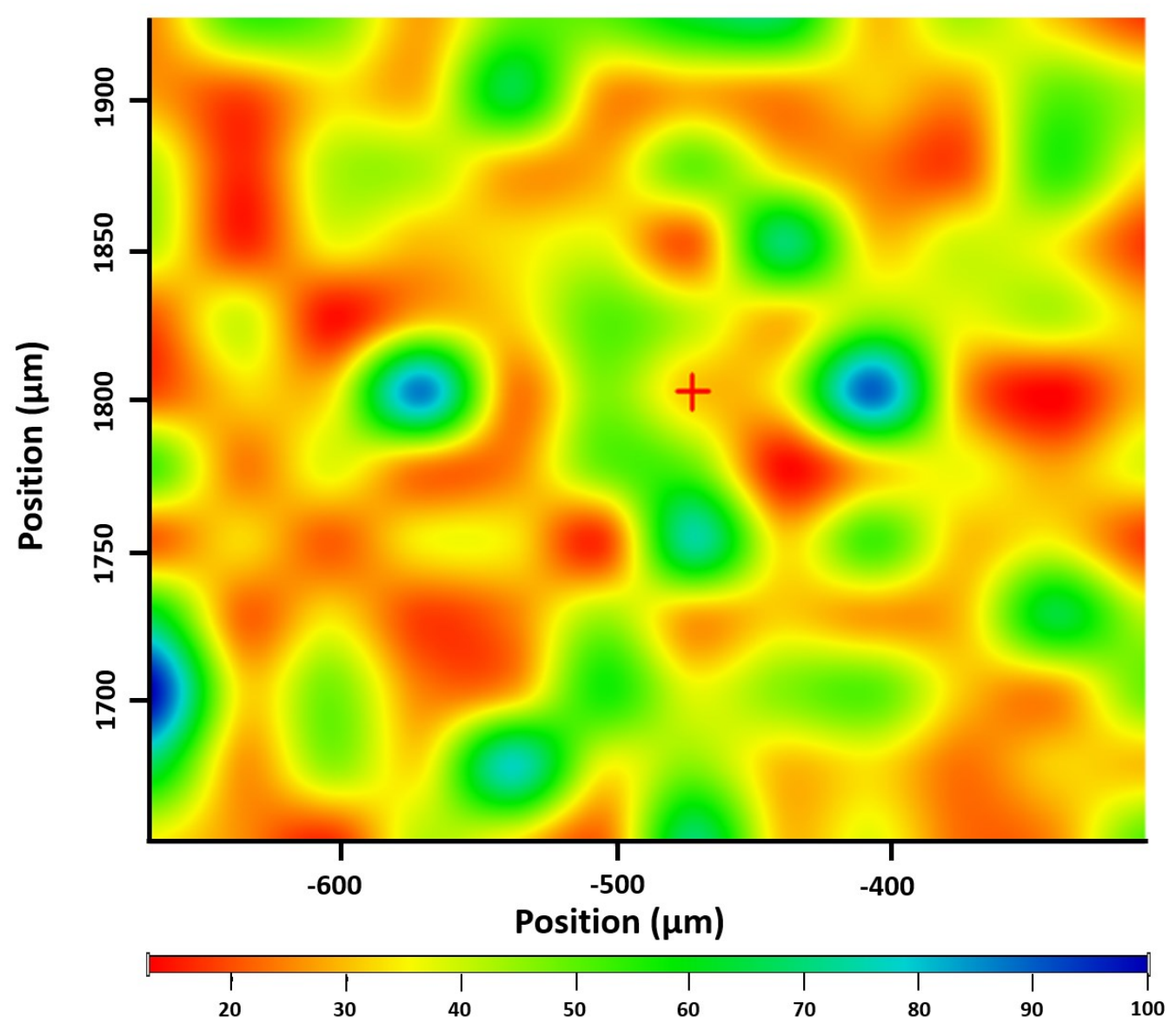



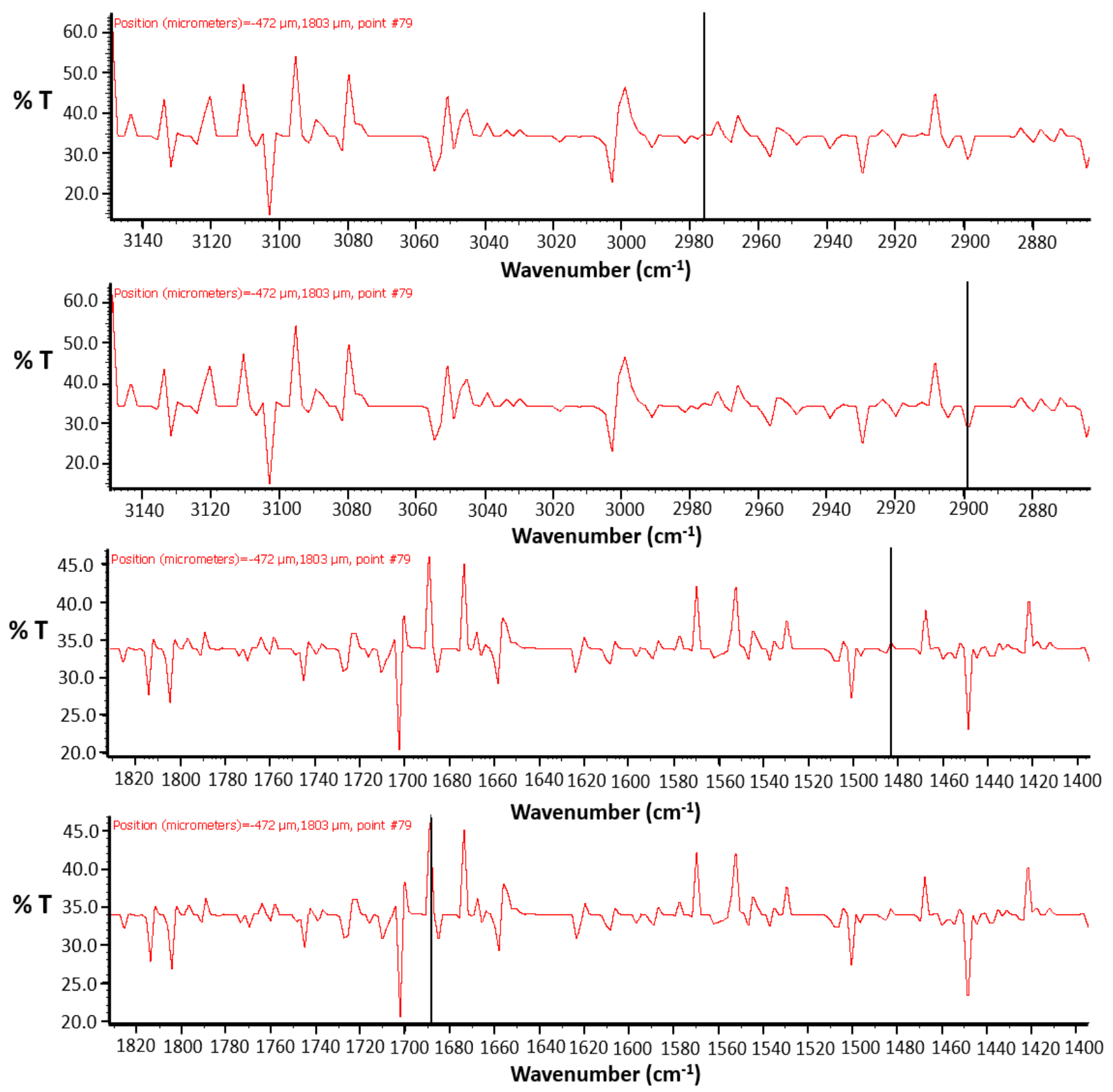

Figure 6. 3D map (top), 2D map (middle) and IR spectra (bottom) of F1 as representative result, 954 


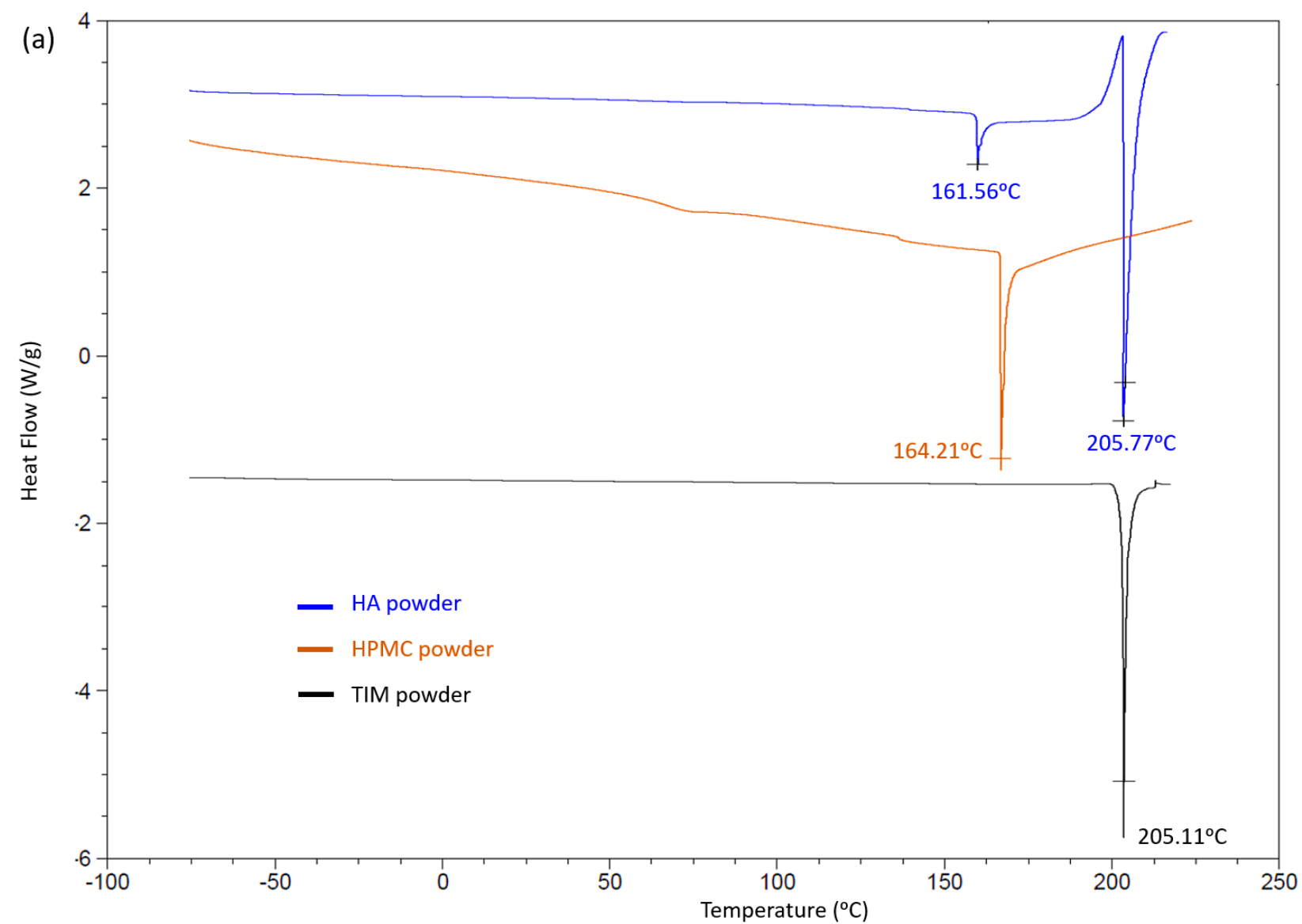

955

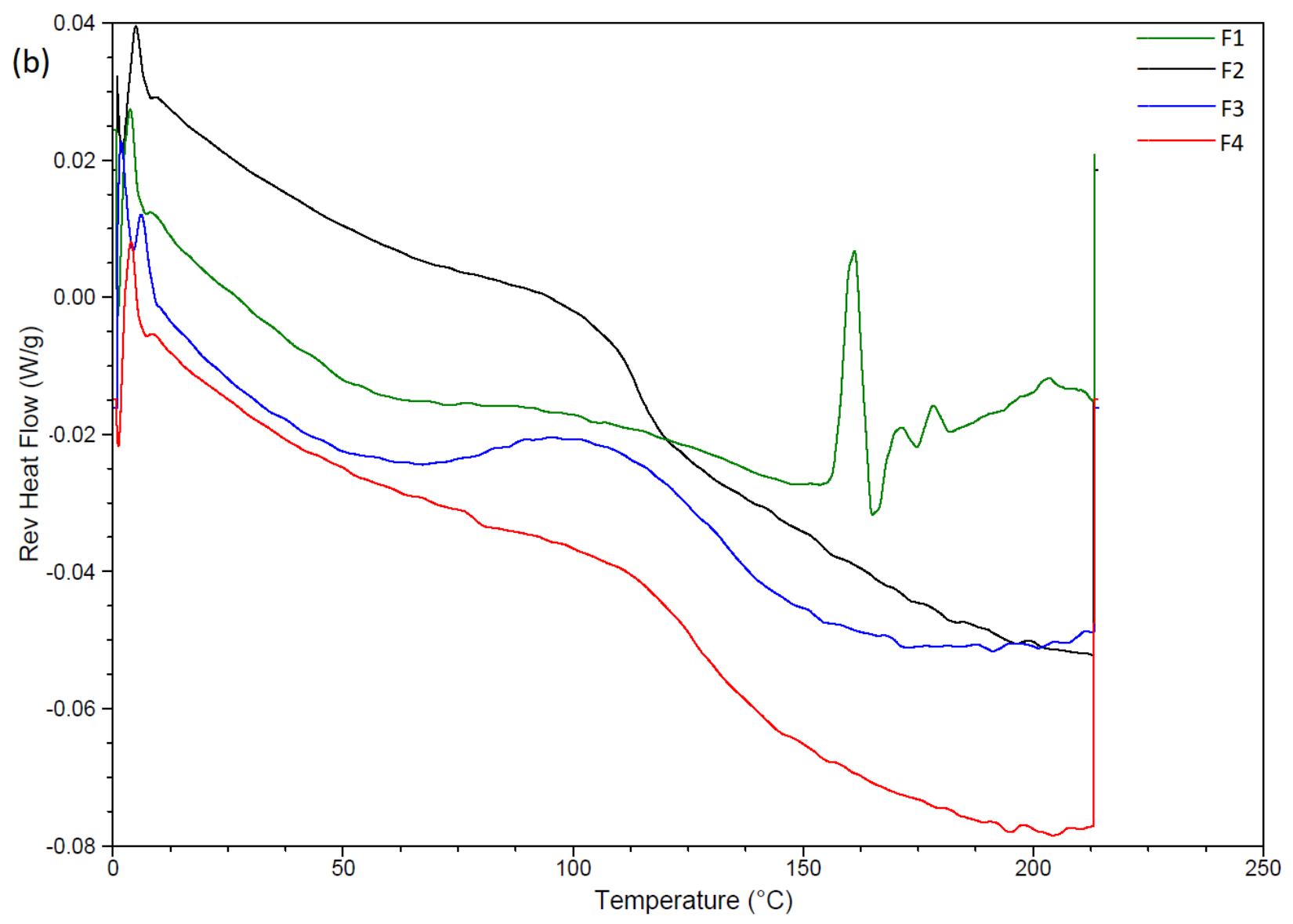

Figure 7. MDSC thermograms of (a) TM powder and (b) formulations F1-F4. 

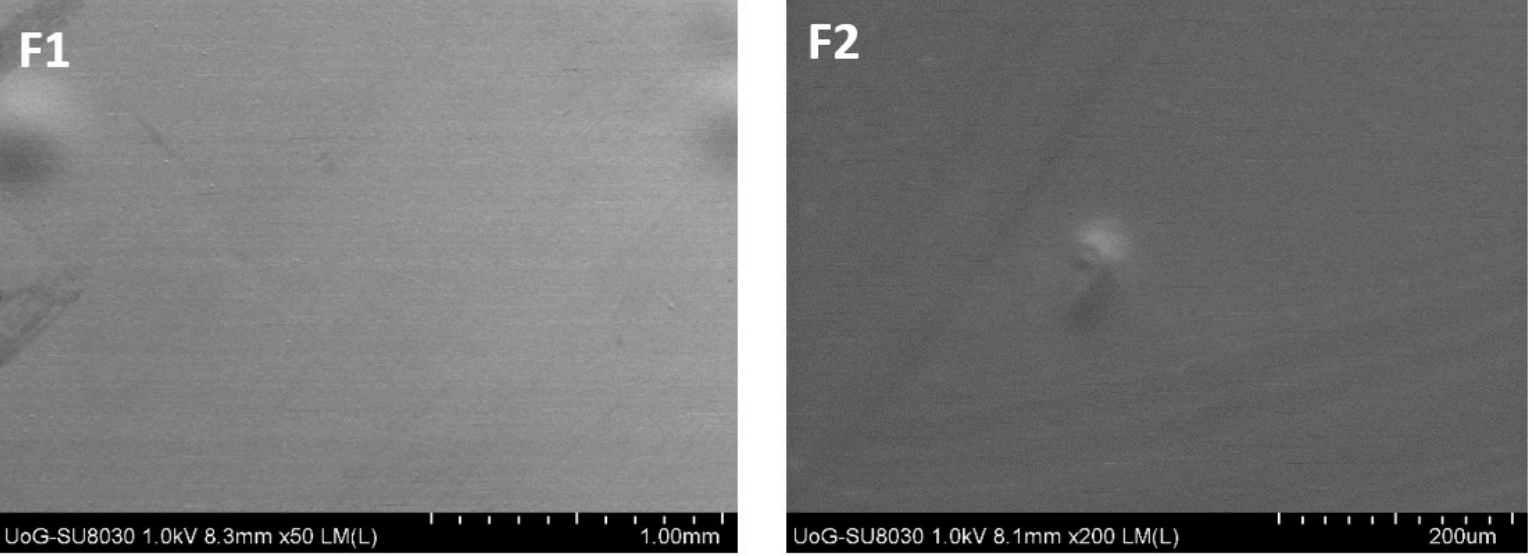

963 Figure 8. SEM micrographs of formulations F1-F4 showing their surface morphology. 


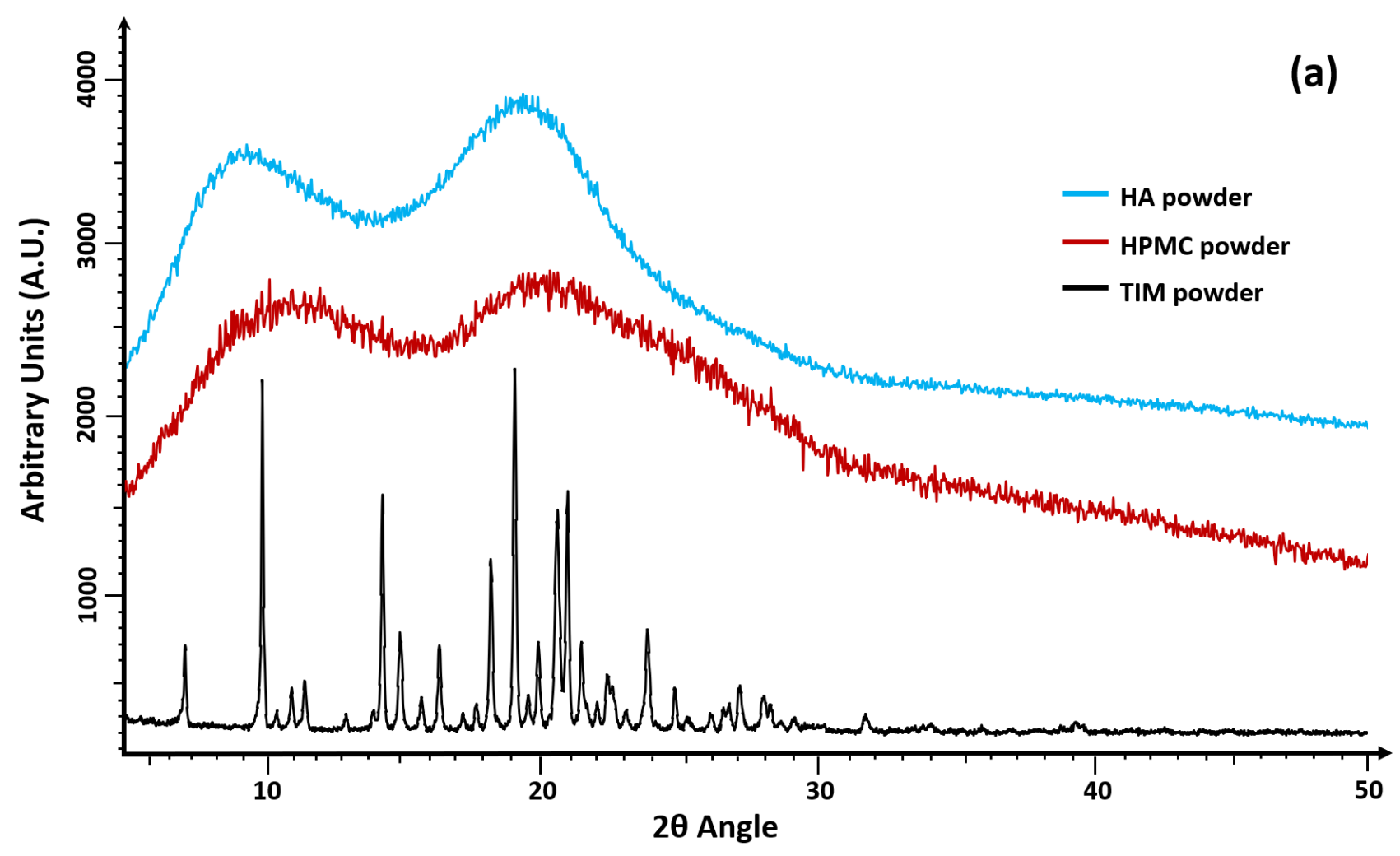

965

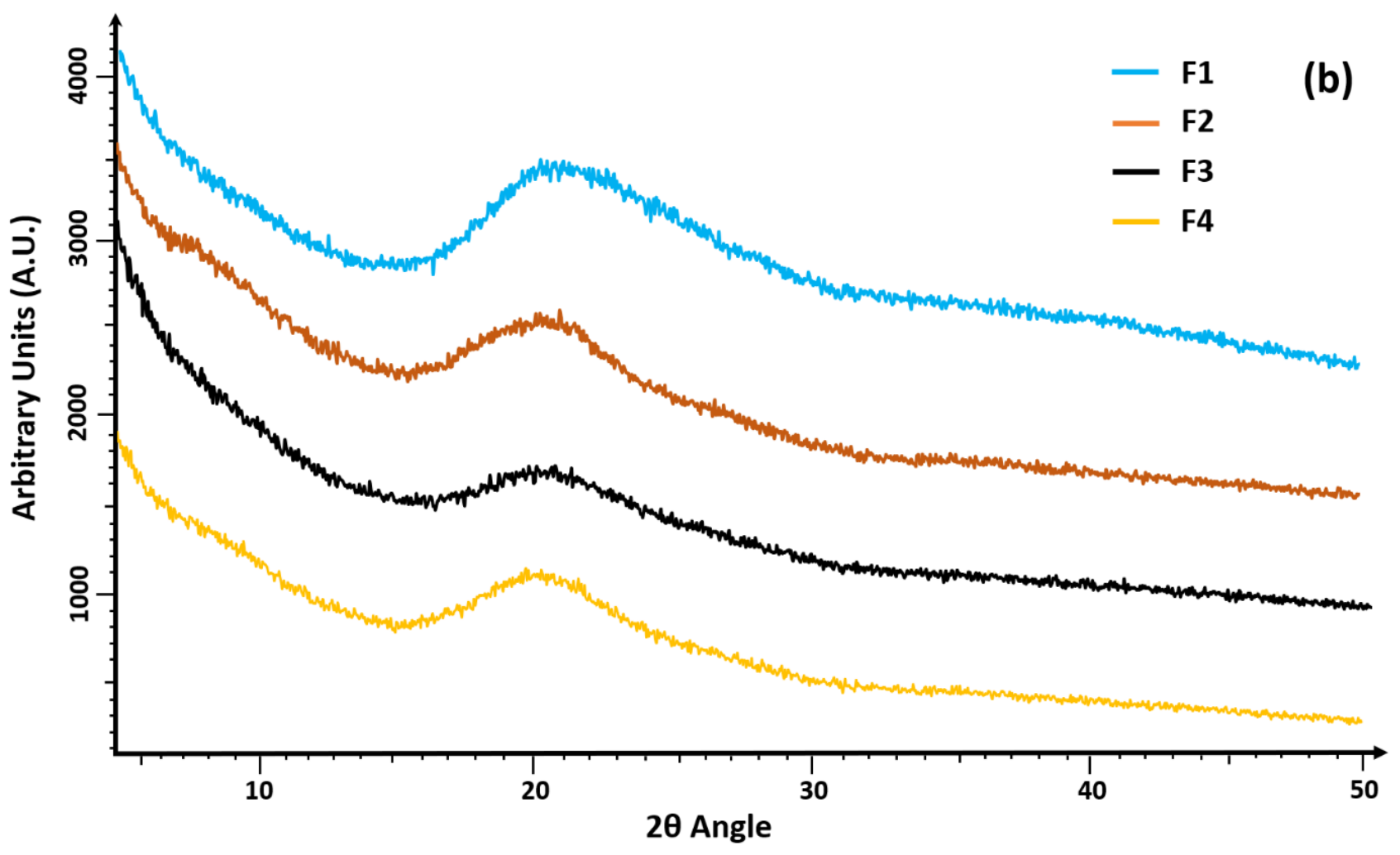

967

Figure 9. XRD diffractograms of (a) TM and polymers' pure powder and (b) formulations F1- 


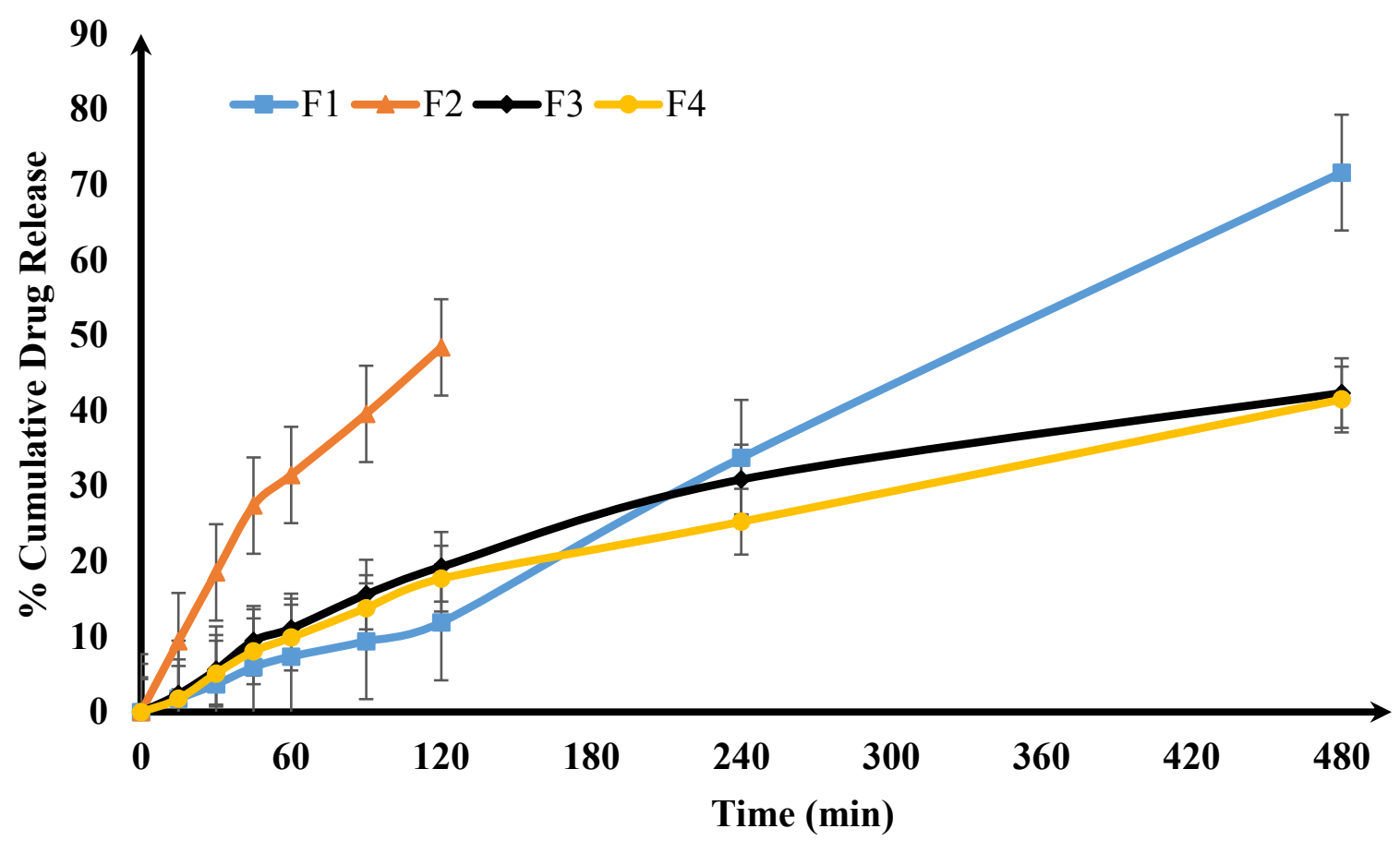

Figure 10. Drug dissolution profiles showing percentage cumulative drug release against time for formulations F1-F4.

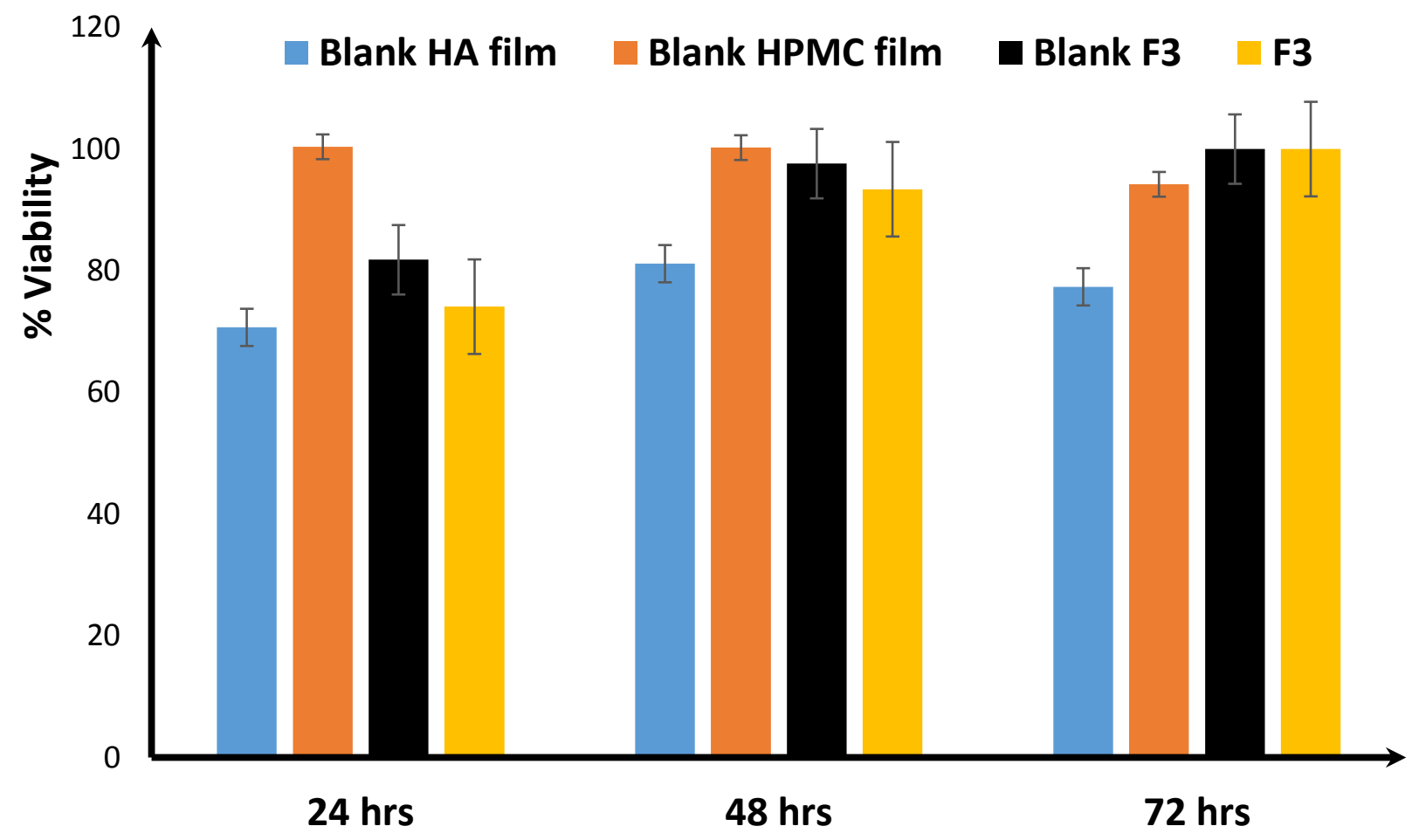

Figure 11. Cell viability of HeLa cells after exposure to the extracts of blank HA film, blank HPMC film, TM loaded composite film (F3) and blank (no TM) F3 films for 24, 48 and 72 hrs (mean $\pm S D, n=6)$. 